\title{
DISCRETE-TIME APPROXIMATIONS OF STOCHASTIC DELAY EQUATIONS: THE MILSTEIN SCHEME
}

\author{
By YaOzhong Hu, ${ }^{1}$ Salah-Eldin A. Mohammed ${ }^{2}$ And Feng Yan \\ University of Kansas, Southern Illinois University and Williams Energy
}

\begin{abstract}
In this paper, we develop a strong Milstein approximation scheme for solving stochastic delay differential equations (SDDEs). The scheme has convergence order 1 . In order to establish the scheme, we prove an infinitedimensional Itô formula for "tame" functions acting on the segment process of the solution of an SDDE. It is interesting to note that the presence of the memory in the SDDE requires the use of the Malliavin calculus and the anticipating stochastic analysis of Nualart and Pardoux. Given the nonanticipating nature of the SDDE, the use of anticipating calculus methods in the context of strong approximation schemes appears to be novel.
\end{abstract}

1. Introduction. Discrete-time strong approximation schemes for stochastic ordinary differential equations (SODEs) are well developed. For an extensive study of these numerical schemes, one may refer to [17], [18] and [19], Chapters 5 and 6 . Some basic ideas of strong and weak orders of convergence are illustrated in [13].

If the rate of change of a physical system depends only on its present state and some noisy input, then the system can often be described by a stochastic ordinary differential equation (SODE). However, in many physical situations the rate of change of the state depends not only on the present but also on the past states of the system. In such cases, stochastic delay differential equations (SDDEs) or stochastic functional differential equations (SFDEs) provide important tools to describe and analyze these systems. For various aspects of the qualitative theory of SFDEs the reader may refer to [20,21] and the references therein.

SDDEs and SFDEs arising in many applications cannot be solved explicitly. Hence, one needs to develop effective numerical techniques for such systems. Depending on the particular physical model, it may be necessary to design strong $L^{p}$ (or almost sure) numerical schemes for pathwise solutions of the underlying SFDE. Strong approximation schemes for SFDEs may be used to

Received April 2001; revised December 2002.

${ }^{1}$ Supported in part by NSF Grants EPS-98-74732, DMS-02-04613 and General Research Fund, University of Kansas.

${ }^{2}$ Supported in part by NSF Grants DMS-97-03596, DMS-99-75462 and DMS-02-03368.

AMS 2000 subject classifications. Primary 34K50, 60H07, 60H35; secondary 60C30, 60H10, 37H10, 34K28.

Key words and phrases. Milstein scheme, Itô's formula, tame functions, anticipating calculus, Malliavin calculus, weak derivatives. 
simulate directly the a.s. stochastic dynamics of their trajectories or their random attractors. SFDEs are used to model population growth with incubation/gestation period [21]. In such models, one is often interested in estimating the actual population rather than its distribution and hence the need for strong approximation schemes.

In this article, we will not consider the order of convergence of weak numerical schemes, although such schemes are useful for some applications of SODEs (see $[13,17]$ and the references therein). In this connection, it is important to note that stochastic systems with memory do not correspond to deterministic PDEs (in finitely many space variables) [20, 21]. Typically, a stochastic system with memory corresponds to an infinite-dimensional Feller diffusion whose principal coefficient degenerates on a hypersurface with finite-codimension ([20], Chapter IV, Theorem 3.2 and [21], Theorem II.3). This aspect of SFDEs is in sharp contrast with the theory of SODEs where the latter theory has traditional ties to diffusions in Euclidean space. In a sense, the numerics of stochastic systems with memory resemble those of SPDEs in one space dimension.

A strong Cauchy-Maruyama scheme for a class of SFDEs with continuous memory, in the context of the Delfour-Mitter state space $\mathbf{R}^{m} \times L^{2}\left([-\tau, 0], \mathbf{R}^{m}\right)$, was developed by Ahmed, Elsanousi and Mohammed [1]. See also [20], page 227, [15] and [4]. As in the case of SODEs, the Cauchy-Maruyama scheme for SFDEs has order of convergence $\frac{1}{2}$ ([20], page 227, [15, 4, 8, 14]).

In Sections 2-5, we establish the strong Milstein scheme for SDDEs with several delays. This scheme has a higher strong order of convergence 1 when compared with the Euler scheme which, as indicated above, has the strong order of convergence 0.5 . Furthermore, when simulating the whole solution path $\{X(t), t \in$ $[0, a]\}$, the Milstein schemes for SDDEs and SODEs have the same complexity, even when one accounts for the simulation of the iterated stochastic integrals in the scheme. (See Appendix B and the remarks therein.) Although the solution of the SDDE is adapted to the (lagged) filtration of the driving noise, methods from anticipating stochastic analysis and the Malliavin calculus are necessary in order to derive an Itô formula for the segment of the solution process. The Itô formula is essential for the development of the Milstein scheme.

In order to put our analysis in proper perspective, we highlight its essential features: (a) The dynamics and the coefficients of the SDDEs are adapted, in fact, driven by Itô integrals; (b) the formulation and implementation of the Milstein scheme do not require anticipating calculus ideas; (c) the proof of convergence of the Milstein scheme as well as the Itô formula employ anticipating calculus techniques; (d) anticipating calculus methods are used in the context of strong approximation schemes rather than weak ones (where the Feynman-Kac formula lends itself naturally to the use of Malliavin calculus methods); (e) the application 
of anticipating calculus methods seems unavoidable as soon as one seeks higherorder approximation results.

In an essentially nonadapted setting, anticipating calculus methods have been used by Pardoux and Protter to study stochastic Volterra equations with anticipating coefficients. See [24] and the references therein. See also [7].

In order to describe our set-up, we need the following notation.

Let $\mathbf{R}^{m}$ be $m$-dimensional Euclidean space with the Euclidean norm $|x|:=$ $\sqrt{x_{1}^{2}+\cdots+x_{m}^{2}}, x=\left(x_{1}, \ldots, x_{m}\right) \in \mathbf{R}^{m}$. Denote $T:=[0, a], J:=[-\tau, 0]$, $C:=C\left(J ; \mathbf{R}^{m}\right)$, where $m$ is a positive integer, $\tau>0$ is a fixed delay [as in (1.6)] and $a>0$. Furnish $C$ with the supremum norm $\|\eta\|_{C}:=\sup _{-\tau \leq s \leq 0}|\eta(s)|$ for all $\eta \in C$.

Define the projection $\Pi: C \rightarrow \mathbf{R}^{m k}$ associated with $s_{1}, \ldots, s_{k} \in[-\tau, 0]$ by

$$
\Pi(\eta):=\left(\eta\left(s_{1}\right), \ldots, \eta\left(s_{k}\right)\right) \in \mathbf{R}^{m k}
$$

for all $\eta \in C$.

Definition 1.1. A function $\Phi \in C\left(T \times C\left(J ; \mathbf{R}^{m}\right) ; \mathbf{R}\right)$ is tame if there exist $\phi \in C\left(T \times \mathbf{R}^{m k}, \mathbf{R}\right)$ and a projection $\Pi: C \rightarrow \mathbf{R}^{m k}$ such that

$$
\Phi(t, \eta)=\phi(t, \Pi(\eta))
$$

for all $t \in T$ and $\eta \in C$.

Let $(\Omega, \mathcal{F}, P)$ be a probability space. For any continuous $m$-dimensional process $X:[-\tau, a] \times \Omega \rightarrow \mathbf{R}^{m}$, define the segment process $X_{t}, t \in[0, a]$, by

$$
X_{t}(u):=X(t+u), \quad t \in[0, a], u \in[-\tau, 0] .
$$

Observe that $\left\{X_{t}\right\}$ may be considered as a $C$-valued or $L^{2}\left(J ; \mathbf{R}^{m}\right)$-valued process.

It is important that one should distinguish between the finite-dimensional current state $X(t)$ and the infinite-dimensional segment $X_{t}, t \in[0, a]$.

Assume that $g: T \times \mathbf{R}^{m k_{1}} \rightarrow L\left(\mathbf{R}^{d} ; \mathbf{R}^{m}\right)$ and $h: T \times \mathbf{R}^{m k_{2}} \rightarrow \mathbf{R}^{m}$ satisfy the following Lipschitz condition:

$$
\begin{aligned}
& |g(t, x)-g(t, y)| \leq L|x-y|, \\
& |h(t, z)-h(t, w)| \leq L|z-w|
\end{aligned}
$$

for all $t \in T, x, y \in \mathbf{R}^{m k_{1}}$ and $z, w \in \mathbf{R}^{m k_{2}}$, where $L>0$ is a constant, together with the boundedness condition

$$
\sup _{0 \leq t \leq a}[|g(t, 0)|+|h(t, 0)|]<\infty .
$$

Let $\Pi_{1}$ and $\Pi_{2}$ be two projections associated with two sets of points $s_{1,1}, \ldots, s_{1, k_{1}} \in[-\tau, 0]$ and $s_{2,1}, \ldots, s_{2, k_{2}} \in[-\tau, 0]$, respectively. Suppose 
$\left\{W(t):=\left(W^{1}(t), \ldots, W^{d}(t)\right): t \geq 0\right\}$ is a $d$-dimensional standard Brownian motion defined on a filtered probability space $\left(\Omega, \mathcal{F},\left(\mathcal{F}_{t}\right)_{t \geq 0}, P\right)$ satisfying the usual conditions. Let $\eta: \Omega \rightarrow C\left([-\tau, 0] ; \mathbf{R}^{m}\right)$ be an $\mathcal{F}_{0}$-measurable initial process.

We will first consider the following class of Itô SDDEs:

$$
X(t)=\left\{\begin{array}{l}
\eta(0)+\int_{0}^{t} g\left(s, \Pi_{1}\left(X_{s}\right)\right) d W(s)+\int_{0}^{t} h\left(s, \Pi_{2}\left(X_{s}\right)\right) d s, \quad t \geq 0, \\
\eta(t), \quad-\tau \leq t<0 .
\end{array}\right.
$$

Under conditions (1.4) and (1.5), the SDDE (1.6) has a unique strong solution (cf. [20], Theorem II.2.1, page 36, and Theorem V.4.3, pages 151 and 152). To see this, let $G(t, \eta):=g\left(t, \Pi_{1}(\eta)\right)$ and $H(t, \eta):=h\left(t, \Pi_{2}(\eta)\right)$ for $t \in[0, a], \eta \in C$. It is easy to check that $G$ and $H$ satisfy the Lipschitz and local boundedness conditions (with respect to the supremum norm on $C$ ) of Theorems II.2.1 and V.4.3 of [20]. Therefore, for each $p \geq 1$, there exists a constant $C=C(p, L, a)>0$ such that

$$
E\left\|X_{t}\right\|_{C}^{2 p} \leq C\left(1+E\|\eta\|_{C}^{2 p}\right)
$$

for all $\eta \in C, t \in[0, a]$.

For any integers $n, l \geq 1$, let $\pi: t_{-l}<t_{-l+1}<\cdots<0=t_{0}<t_{1}<t_{2}<\cdots<t_{n}$ be a partition of $[-\tau, a]$. Denote by $|\pi|:=\max _{-l \leq i \leq n-1}\left(t_{i+1}-t_{i}\right)$, the mesh of $\pi$. We now introduce the following Milstein scheme for the SDDE (1.6):

$$
\begin{aligned}
X^{i, \pi}(t)= & X^{i, \pi}\left(t_{k}\right)+h^{i}\left(t_{k}, \Pi_{2}\left(X_{t_{k}}^{\pi}\right)\right)\left(t-t_{k}\right) \\
+ & g^{i j}\left(t_{k}, \Pi_{1}\left(X_{t_{k}}^{\pi}\right)\right)\left(W^{j}(t)-W^{j}\left(t_{k}\right)\right) \\
+ & \frac{\partial g^{i j}}{\partial x_{i_{1} j_{1}}}\left(t_{k}, \Pi_{1}\left(X_{t_{k}}^{\pi}\right)\right) u^{i_{1} j_{1}, \pi}\left(t_{k}+s_{1, j_{1}}\right) \\
& \quad \times I_{j, j_{1}}\left(t_{k}+s_{1, j_{1}}, t+s_{1, j_{1}} ; s_{1, j_{1}}\right)
\end{aligned}
$$

for $t_{k}<t \leq t_{k+1}$, and

$$
X^{\pi}(t):=\eta^{\pi}(t), \quad t \in[-\tau, 0],
$$

where

$$
\begin{gathered}
u^{i_{1} j_{1}, \pi}(t):= \begin{cases}g^{i_{1} j_{1}}\left(t, \Pi_{1}\left(X_{t}^{\pi}\right)\right), & t \geq 0, \\
0, & -\tau \leq t<0,\end{cases} \\
I_{j, j_{1}}\left(t_{0}+s, t+s ; s\right):=\int_{t_{0}}^{t} \int_{t_{0}+s}^{t_{1}+s} \circ d W^{j}\left(t_{2}\right) \circ d W^{j_{1}}\left(t_{1}\right), \\
\quad t \geq t_{0} \geq 0, s \in[-\tau, 0],
\end{gathered}
$$

and the starting path $\eta^{\pi} \in C\left(J, \mathbf{R}^{m}\right)$ is prescribed (e.g., a piecewise linear approximation of $\eta$ using the partition points $\left.\left\{t_{-l}, \ldots, t_{0}\right\}\right)$. In (1.8), $X^{i}, h^{i}$ and $g^{i j}$ 
denote coordinate representations of $X, h$ and $g$ with respect to standard bases in the underlying Euclidean spaces, and the Einstein summation convention is used for repeated indices.

In order to establish strong convergence of the above Milstein scheme for the SDDE (1.6), it turns out, surprisingly, that one requires the use of anticipating calculus techniques developed by Nualart and Pardoux [23]. In particular, one needs to develop an infinite-dimensional Itô formula for "tame" functions acting on the segment $X_{t}$ of the solution $X$ of (1.6). Such an Itô formula is given in Section 2, Theorem 2.3. The formula is proved via anticipating calculus methods [23]. To understand the need for anticipating calculus in such an intrinsically adapted setting, it is instructive to look at the following simple one-dimensional SDDE:

$$
\begin{aligned}
d X(t) & =g(X(t-1), X(t)) d W(t), \quad t \geq 0, \\
X(t) & =W(t), \quad-1 \leq t<0,
\end{aligned}
$$

where $g: \mathbf{R}^{2} \rightarrow \mathbf{R}$ is a smooth function and $W(t), t \geq-1$, is a one-dimensional Brownian motion. For a second-order scheme, we formally seek a stochastic differential of the coefficient $g(X(t-1), X(t))$ on the right-hand side of the above SDDE. For $t \in(0,1]$, this gives formally

$$
\begin{aligned}
d\{g(X(t-1), X(t))\} & \\
= & d\{g(W(t-1), X(t))\} \\
= & \frac{\partial g}{\partial x}(W(t-1), X(t)) d W(t-1) \\
& +\frac{\partial g}{\partial y}(W(t-1), X(t)) g(X(t-1), X(t)) d W(t) \\
& + \text { second-order terms. }
\end{aligned}
$$

Note that although the coefficient $g(X(t-1), X(t))$ is $\mathcal{F}_{t}$-measurable, the first term $\frac{\partial g}{\partial x}(W(t-1), X(t)) d W(t-1)$ in the right-hand side of the last equality is an anticipating differential. Furthermore, it appears that the $\left(\mathcal{F}_{t}\right)_{0 \leq t \leq 1}$-adapted process $[0,1] \ni t \rightarrow(X(t-1), X(t)) \in \mathbf{R}^{2}$ is not a semimartingale with respect to any natural filtration. In addition to this difficulty, the components $X(t-1)$ and $X(t)$ are not independent, so the existing anticipating versions of Itô's formula do not apply (cf. [2, 3] and [23]); hence the need for a new Itô formula for tame functions in order to justify the above computation. In Theorem 2.1 in the next section we establish such a formula.

Using the above-mentioned Itô formula and appropriate estimates on the weak Cameron-Martin derivatives of $X$, it is shown in Section 5 (Theorem 5.2) that, under suitable regularity conditions on the coefficients of (1.6), one gets the following global error estimate for the Milstein approximations:

$$
E \sup _{0 \leq t \leq a}\left\|X_{t}^{\pi}-X_{t}\right\|_{C}^{q} \leq C(q)|\pi|^{q}
$$


for any $q \geq 1$. This says that the Milstein scheme has strong order of convergence 1 .

2. Itô's formula for "tame" functions. In order to derive higher-order numerical schemes for SDDEs, we shall first prove an Itô formula for "tame" functions on $C\left(J, \mathbf{R}^{m}\right)$ (Definition 1.1).

Suppose that $W(t):=\left(W^{1}(t), \ldots, W^{d}(t)\right), t \geq 0$, is $d$-dimensional standard Brownian motion on a filtered probability space $\left(\Omega, \mathcal{F},\left(\mathcal{F}_{t}\right)_{t \geq 0}, P\right)$. Denote by $D=\left(D_{1}, \ldots, D_{d}\right)$ the Malliavin differentiation operator associated with $\{W(t): t \geq 0\}$. Assume

$$
X(t)= \begin{cases}\eta(0)+\int_{0}^{t} u(s) d W(s)+\int_{0}^{t} v(s) d s, & t>0, \\ \eta(t), & -\tau \leq t \leq 0,\end{cases}
$$

where $\eta$ belongs to $C$ and is of bounded variation, $u=\left(u^{1}, \ldots, u^{m}\right)^{T}, u^{i} \in \mathbb{L}_{d, \text { loc }}^{2,4}$, $v=\left(v^{1}, \ldots, v^{m}\right)^{T}$, and $v^{i} \in \mathbb{L}_{\text {loc }}^{1,4}$. One can refer to ([22], pages 61, 151 and 161), for the definition of $\mathbb{L}_{d}^{k, p}$. Note that the processes $u$ and $v$ may not be adapted to the Brownian filtration $\left(\mathcal{F}_{t}\right)_{t \geq 0}$. For convenience, we define $u(t)=0$ for $t<0$ or $t>a$,

$$
v(t)= \begin{cases}0, & t>a, \\ \eta^{\prime}(t), & -\tau \leq t \leq 0 .\end{cases}
$$

We also set $W(t)=0$ if $t<0$ or $t>a$, and denote

$$
\begin{aligned}
U(t) & :=\int_{0}^{t} u(s) d W(s), \\
V(t) & := \begin{cases}\eta(0)+\int_{0}^{t} v(s) d s, & t>0, \\
\eta(t), & -\tau \leq t \leq 0 .\end{cases}
\end{aligned}
$$

If $u \in \mathbb{L}_{\text {loc }}^{2, p}$ for some $p>4$, then the indefinite Skorohod integral $\int_{0}^{t} u(s) d W(s)$ has a continuous version. Hence, we may assume that the process $X(t), t \geq-\tau$, is sample continuous.

Let $T=[0, a], J=[-\tau, 0], C=C\left(J, \mathbf{R}^{m}\right)$ be as before, and let $\Pi$ be the projection associated with $s_{1}, \ldots, s_{k} \in J$. Although there is a multidimensional Itô formula for $\phi(t, X(t))$ ([2,3] and [22]), we cannot apply it to $\phi\left(t, \Pi\left(X_{t}\right)\right)$ because $\Pi\left(U_{t}\right)$ is of the form

$$
\left(\int_{0}^{t} u\left(s+s_{1}\right) d W\left(s+s_{1}\right), \ldots, \int_{0}^{t} u\left(s+s_{k}\right) d W\left(s+s_{k}\right)\right), \quad t>0
$$

and the components of the $d k$-dimensional process $\left(W\left(t+s_{1}\right), \ldots, W\left(t+s_{k}\right)\right)$ are not independent. However, the ideas in [23], Section 6, and in [22], page 161, can be used to derive an Itô formula for $\phi\left(t, \Pi\left(X_{t}\right)\right)$. See [28] for further details. 
We denote by

$$
\delta_{i j}= \begin{cases}1, & i=j \\ 0, & i \neq j\end{cases}
$$

the Kronecker delta.

For any process $X(t), t \in[-\tau, a]$, denote its (delayed) increments by

$$
\Delta_{l i} X:=X\left(t_{l}+s_{i}\right)-X\left(t_{l-1}+s_{i}\right), \quad 1 \leq l \leq n, i=1,2, \ldots, k .
$$

Assume that $\phi \in C^{1,2}\left(T \times \mathbf{R}^{m k}, \mathbf{R}\right)$, and write

$$
\phi(t, \vec{x}):=\phi\left(t, \vec{x}_{1}, \ldots, \vec{x}_{m}\right)
$$

where $\vec{x}:=\left(\vec{x}_{1}, \ldots, \vec{x}_{m}\right), \vec{x}_{i}:=\left(x_{i 1}, \ldots, x_{i k}\right) \in \mathbf{R}^{k}, 1 \leq i \leq m$.

We now state an Itô formula for "tame" functions.

THEOREM 2.1. Assume that $X$ is a continuous process defined by (2.1), where $\eta: J \rightarrow \mathbf{R}^{m}$ is of bounded variation, $u=\left(u^{1}, \ldots, u^{m}\right)^{T}, u^{i} \in \mathbb{L}_{d, \text { loc }}^{2,4}$, $v=\left(v^{1}, \ldots, v^{m}\right)^{T}$ and $v^{i} \in \mathbb{L}_{\text {loc }}^{1,4}$. Suppose $\phi \in C^{1,2}\left(T \times \mathbf{R}^{m k}, \mathbf{R}\right)$. Then

$$
\begin{gathered}
\phi\left(t, \Pi\left(X_{t}\right)\right)-\phi\left(0, \Pi\left(X_{0}\right)\right) \\
=\int_{0}^{t} \frac{\partial \phi}{\partial s}\left(s, \Pi\left(X_{s}\right)\right) d s+\int_{0}^{t} \frac{\partial \phi}{\partial \vec{x}}\left(s, \Pi\left(X_{s}\right)\right) d\left(\Pi\left(X_{s}\right)\right) \\
+\frac{1}{2} \sum_{i, j=1}^{k} \sum_{i_{1}, j_{1}=1}^{m} \int_{0}^{t} \frac{\partial^{2} \phi}{\partial x_{i_{1} i} \partial x_{j_{1} j}}\left(s, \Pi\left(X_{s}\right)\right) u^{i_{1}}\left(s+s_{i}\right) \\
\times D_{s+s_{i}} X^{j_{1}}\left(s+s_{j}\right) d s .
\end{gathered}
$$

\section{REMARKS.}

1. The Itô formula (2.7) may also be expressed in the form

$$
\begin{aligned}
\phi\left(t, \Pi\left(X_{t}\right)\right)-\phi\left(0, \Pi\left(X_{0}\right)\right) \\
=\int_{0}^{t} \frac{\partial \phi}{\partial s}\left(s, \Pi\left(X_{s}\right)\right) d s+\int_{0}^{t} \frac{\partial \phi}{\partial \vec{x}}\left(s, \Pi\left(X_{s}\right)\right) d\left(\Pi\left(X_{s}\right)\right) \\
\quad+\frac{1}{2} \sum_{i, j=1}^{k} \int_{0}^{t} \operatorname{Tr}\left[\frac{\partial^{2} \phi}{\partial \vec{x}_{i} \partial \vec{x}_{j}}\left(s, \Pi\left(X_{s}\right)\right)\left(\Theta_{s}\left(s_{i}, s_{j}\right)\right)\right] d s,
\end{aligned}
$$

where

$$
\Theta_{S}(\alpha, \beta):=\frac{1}{2}\left\{(u \Lambda)_{S} X_{S}(\alpha, \beta)+(u \Lambda)_{S} X_{S}(\beta, \alpha)\right\}, \quad \alpha, \beta \in[-\tau, 0]
$$


and the two-parameter process $(u \Lambda)_{s} X_{s}: \Omega \times J^{2} \rightarrow L\left(\mathbf{R}^{m} ; \mathbf{R}^{m}\right)$ is defined by

$$
\begin{aligned}
& (u \Lambda)_{s} X_{s}(\alpha, \beta) \\
& :=I_{\{0 \leq s+\alpha \wedge \beta\}} u(s+\alpha) \\
& \quad \times\left[u^{T}(s+\alpha) I_{\{0 \leq s+\alpha \leq s+\beta\}}\right. \\
& \left.\quad+\int_{0}^{s+\beta} D_{s+\alpha} u(r) d W(r)+\int_{0}^{s+\beta} D_{s+\alpha} v(r) d r\right]
\end{aligned}
$$

for all $\alpha, \beta \in[-\tau, 0]$.

2. Suppose $d=m=1$. Let us define a trace operator $\nabla$. For $1 \leq i, j \leq k$, define

$$
\nabla_{s_{i}, s_{j}}^{ \pm} X(s):=\lim _{\varepsilon \downarrow 0}\left(D_{s+s_{i}} X\left(s+s_{j}+\varepsilon\right) \pm D_{s+s_{i}} X\left(s+s_{j}-\varepsilon\right)\right) \in \mathbf{R}
$$

and $\nabla_{s_{i}}^{ \pm} X(s):=\left(\nabla_{s_{i}, s_{1}}^{ \pm} X(s), \ldots, \nabla_{s_{i}, s_{k}}^{ \pm} X(s)\right) \in \mathbf{R}^{k}$. Then the Itô formula for "tame" functions can be written as

$$
\begin{aligned}
\phi\left(t, \Pi\left(X_{t}\right)\right)-\phi\left(0, \Pi\left(X_{0}\right)\right) \\
=\int_{0}^{t} \frac{\partial \phi}{\partial s}\left(s, \Pi\left(X_{s}\right)\right) d s+\int_{0}^{t} \frac{\partial \phi}{\partial \vec{x}}\left(s, \Pi\left(X_{s}\right)\right) d \Pi\left(W_{s}\right) \\
\quad+\frac{1}{2} \sum_{i=1}^{k} \int_{0}^{t}\left\langle\frac{\partial^{2} \phi}{\partial x_{i}^{2}}\left(s, \Pi\left(X_{s}\right)\right) \nabla_{s_{i}}^{+} X(s), \nabla_{s_{i}}^{-} X(s)\right\rangle_{\mathbf{R}^{d}} d s
\end{aligned}
$$

a.s. for all $t \in T$, where $\vec{x}:=\left(x_{1}, \ldots, x_{k}\right)$ and $\langle\cdot, \cdot\rangle_{\mathbf{R}^{d}}$ denotes the Euclidean inner product on $\mathbf{R}^{d}$. See [23], Remark 7.6.

3. The Itô formula (2.7) still holds if the initial path is an $\mathcal{F}_{0}$-measurable process $\eta: \Omega \rightarrow C$ with a.a. sample paths of bounded variation. A similar remark also holds for Theorem 5.2 of Section 5 .

For simplicity, we shall prove the Itô formula for the case $d=m=1$. We thus assume in what follows that $d=m=1$.

Proof OF THEOREM 2.1. For any integer $n \geq 1$, let $\left\{\pi_{n}: 0=t_{0}<t_{1}<\cdots<\right.$ $\left.t_{n}=a\right\}$ be a partition of $[0, a]$. Then by Taylor's theorem, we may write

$$
\begin{aligned}
\phi\left(t, \Pi\left(X_{t}\right)\right)-\phi\left(0, \Pi\left(X_{0}\right)\right) & \\
=\sum_{l=1}^{n} & {\left[\phi\left(t_{l}, \Pi\left(X_{t_{l}}\right)\right)-\phi\left(t_{l-1}, \Pi\left(X_{t_{l}}\right)\right)\right] } \\
& \quad+\left[\phi\left(t_{l-1}, \Pi\left(X_{t_{l}}\right)\right)-\phi\left(t_{l-1}, \Pi\left(X_{t_{l-1}}\right)\right)\right]
\end{aligned}
$$




$$
\begin{aligned}
= & \sum_{l=1}^{n} \frac{\partial \phi}{\partial s}\left(\hat{t}_{l}, \Pi\left(X_{t_{l}}\right)\right) \Delta t_{l} \\
& +\sum_{l=1}^{n}\left\{\sum_{i=1}^{k} \frac{\partial \phi}{\partial x_{i}}\left(t_{l-1}, \Pi\left(X_{t_{l-1}}\right)\right) \Delta_{l i} X\right. \\
& \left.+\frac{1}{2} \sum_{i, j=1}^{k} \frac{\partial^{2} \phi}{\partial x_{i} \partial x_{j}}\left(t_{l-1}, \Pi\left(\bar{X}_{t_{l}}\right)\right) \Delta_{l i} X \Delta_{l j} X\right\}, \quad t \in T,
\end{aligned}
$$

where

$$
\Delta t_{l}:=t_{l}-t_{l-1}, \quad \bar{X}_{t_{l}}=X_{t_{l-1}}+\alpha_{l}\left(X_{t_{l}}-X_{t_{l-1}}\right), \quad \hat{t}_{l}=t_{l-1}+\gamma_{l} \Delta t_{l}
$$

for some random variables $0 \leq \alpha_{l}, \gamma_{l} \leq 1, l=1, \ldots, n$. The Itô formula (2.10) will then follow from Propositions 2.3 and 2.4.

The rest of this section is devoted to the proofs of Propositions 2.2-2.4.

Proposition 2.2. Suppose that $W(t)$ is a one-dimensional Brownian motion. Let $u \in \mathbb{L}_{\mathrm{loc}}^{1,2}$ be such that $u(t)=0$ if $t>$ a or $t<0$. Assume that $-\tau \leq s_{1}$, $s_{2} \leq 0$ and let $\pi_{n}: 0=t_{0}<t_{1}<\cdots<t_{n}=a$ be a family of partitions of $T=[0, a]$, with $\left|\pi_{n}\right| \rightarrow 0$ as $n \rightarrow \infty$. Then

$$
\lim _{n \rightarrow \infty}\left[\sum_{l=1}^{n} \int_{t_{l-1}+s_{1}}^{t_{l}+s_{1}} u(s) d W(s)\right]^{2}=\int_{0}^{a+s_{1}} u^{2}(s) d s
$$

in probability. If $s_{1} \neq s_{2}$, then

$$
\lim _{n \rightarrow \infty} \sum_{l=1}^{n} \int_{t_{l-1}+s_{1}}^{t_{l}+s_{1}} u(s) d W(s) \int_{t_{l-1}+s_{2}}^{t_{l}+s_{2}} u(s) d W(s)=0
$$

in probability. Furthermore, if $u \in \mathbb{L}^{1,2}$, then the above convergences are in $L^{1}(\Omega, \mathbf{R})$.

PROOF. We prove the proposition for $u \in \mathbb{L}^{1,2}$. The general case $u \in \mathbb{L}_{\text {loc }}^{1,2}$ follows by a standard localization argument [22].

If $u_{i}, u_{j}, v_{i}, v_{j} \in \mathbb{L}^{1,2}$ with $u_{i}(t)=v_{i}(t)=0$ if $t<0$ or $t>a+s_{i}$ and $u_{j}(t)=v_{j}(t)=0$ if $t<0$ or $t>a+s_{j}$. Set

$$
\begin{array}{rlrl}
U_{i}(t) & :=\int_{0}^{t} u_{i}(s) d W(s), & V_{i}(t) & :=\int_{0}^{t} v_{i}(s) d W(s), \\
U_{j}(t) & :=\int_{0}^{t} u_{j}(s) d W(s), & V_{j}(t):=\int_{0}^{t} v_{j}(s) d W(s) .
\end{array}
$$


Then

$$
\begin{aligned}
E\left|\sum_{l=1}^{n} \Delta_{l i} U_{i} \Delta_{l j} U_{j}-\sum_{l=1}^{n} \Delta_{l i} V_{i} \Delta_{l j} V_{j}\right| \\
=E\left|\sum_{l=1}^{n} \Delta_{l i}\left(U_{i}-V_{i}\right) \Delta_{l j} U_{j}+\sum_{l=1}^{n} \Delta_{l i} V_{i} \Delta_{l j}\left(U_{j}-V_{j}\right)\right| \\
\leq E\left|\sum_{l=1}^{n} \Delta_{l i}\left(U_{i}-V_{i}\right) \Delta_{l j} U_{j}\right|+E\left|\sum_{l=1}^{n} \Delta_{l i} V_{i} \Delta_{l j}\left(U_{j}-V_{j}\right)\right| \\
\leq\left(E \sum_{l=1}^{n}\left|\Delta_{l i}\left(U_{i}-V_{i}\right)\right|^{2}\right)^{1 / 2}\left(E \sum_{l=1}^{n}\left|\Delta_{l j}\left(U_{j}\right)\right|^{2}\right)^{1 / 2} \\
\quad+\left(E \sum_{l=1}^{n}\left|\Delta_{l i}\left(V_{i}\right)\right|^{2}\right)^{1 / 2}\left(E \sum_{l=1}^{n}\left|\Delta_{l j}\left(U_{j}-V_{j}\right)\right|^{2}\right)^{1 / 2} .
\end{aligned}
$$

By an $L^{p}$ estimate of the Skorohod integral ([23], Proposition 3.5, and [22], page 158), we have

$$
\begin{aligned}
E \sum_{l=1}^{n}\left|\Delta_{l j} U_{j}\right|^{2} & \\
= & E \sum_{l=1}^{n}\left|\int_{t_{l-1}+s_{j}}^{t_{l}+s_{j}} u_{j}(s) d W(s)\right|^{2} \\
= & E \sum_{l=1}^{n}\left|\int_{0}^{a} I_{\left(t_{l-1}+s_{j}, t_{l}+s_{j}\right]}(s) u_{j}(s) d W(s)\right|^{2} \\
\leq & \sum_{l=1}^{n} \int_{0}^{a} I_{\left(t_{l-1}+s_{j}, t_{l}+s_{j}\right]}(s) E u_{j}^{2}(s) d s \\
& +\sum_{l=1}^{n} \int_{0}^{a} \int_{0}^{a} I_{\left(t_{l-1}+s_{j}, t_{l}+s_{j}\right]}(s) E\left(D_{t} u u_{j}(s)\right)^{2} d s d t \\
= & \int_{0}^{a} E u_{j}^{2}(s) d s+\int_{0}^{a} \int_{0}^{a} E\left(D_{t} u_{j}(s)\right)^{2} d s d t \\
= & \left\|u_{j}\right\|_{1,2}^{2} .
\end{aligned}
$$

Hence we obtain the following inequality:

$$
\begin{aligned}
& E\left|\sum_{l=1}^{n} \Delta_{l i} U_{i} \Delta_{l j} U_{j}-\sum_{l=1}^{n} \Delta_{l i} V_{i} \Delta_{l j} V_{j}\right| \\
& \quad \leq\left\|u_{i}-v_{i}\right\|_{1,2}\left\|u_{j}\right\|_{1,2}+\left\|v_{i}\right\|_{1,2}\left\|u_{j}-v_{j}\right\|_{1,2} .
\end{aligned}
$$


Since $\mathbb{L}^{1,2} \cap L^{4}(\Omega \times[0, a])$ is dense in $\mathbb{L}^{1,2}$, it suffices to prove (2.12) for the case $u \in \mathbb{L}^{1,2} \cap L^{4}(\Omega \times[0, a])$. Set

$$
u_{i}(t):= \begin{cases}u(t), & 0 \leq t \leq a+s_{i} \\ 0, & t<0 \text { or } t>a+s_{i}\end{cases}
$$

Define

$$
u_{i}^{n}(t):=\sum_{l=1}^{n} \frac{I_{\left(t_{l-1}+s_{i}, t_{l}+s_{i}\right]}(t)}{t_{l}-t_{l-1}} \int_{t_{l-1}+s_{i}}^{t_{l}+s_{i}} u(s) d s
$$

and $u_{j}^{n}$ similarly. Let

$$
U_{i}(t):=\int_{0}^{t} u_{i}(s) d W(s), \quad U_{i}^{n}(t):=\int_{0}^{t} u_{i}^{n}(s) d W(s),
$$

$$
U_{j}(t):=\int_{0}^{t} u_{j}(s) d W(s), \quad V_{j}^{n}(t):=\int_{0}^{t} u_{j}^{n}(s) d W(s) .
$$

Using (2.14) it is easy to check that

$$
\lim _{n \rightarrow \infty} E\left|\sum_{l=1}^{n} \Delta_{l i} U_{i}^{n} \Delta_{l j} U_{j}^{n}-\sum_{l=1}^{n} \Delta_{l i} U_{i} \Delta_{l j} U_{j}\right|=0 .
$$

By the formula for the Skorohod integral of a process multiplied by a random variable ([23], Theorem 3.2), we get

$$
\begin{aligned}
\Delta_{l i} U_{i}^{n}= & \int_{t_{l-1}+s_{i}}^{t_{l}+s_{i}} \sum_{k=1}^{n} \frac{I_{\left(t_{k-1}+s_{i}, t_{k}+s_{i}\right]}(t)}{t_{k}-t_{k-1}} \int_{t_{k-1}+s_{i}}^{t_{k}+s_{i}} u_{i}(s) d s d W(t) \\
= & \frac{1}{t_{l}-t_{l-1}} \int_{t_{l-1}+s_{i}}^{t_{l}+s_{i}} u_{i}(s) d s\left[W\left(t_{l}+s_{i}\right)-W\left(t_{l-1}+s_{i}\right)\right] \\
& +\frac{1}{t_{l}-t_{l-1}} \int_{t_{l-1}+s_{i}}^{t_{l}+s_{i}} \int_{t_{l-1}+s_{i}}^{t_{l}+s_{i}} D_{t} u_{i}(s) d s d t \\
= & P_{l i} \Delta_{l i} W+Q_{l i},
\end{aligned}
$$

where

$$
\begin{aligned}
P_{l i} & :=\frac{1}{t_{l}-t_{l-1}} \int_{t_{l-1}+s_{i}}^{t_{l}+s_{i}} u_{i}(s) d s \\
Q_{l i} & :=\frac{1}{t_{l}-t_{l-1}} \int_{t_{l-1}+s_{i}}^{t_{l}+s_{i}} \int_{t_{l-1}+s_{i}}^{t_{l}+s_{i}} D_{t} u_{i}(s) d s d t
\end{aligned}
$$


Therefore,

$$
\begin{aligned}
\sum_{l=1}^{n} \Delta_{l i} U_{i}^{n} \Delta_{l j} U_{j}^{n}= & \sum_{l=1}^{n}\left(P_{l i} \Delta_{l i} W+Q_{l i}\right)\left(P_{l j} \Delta_{l j} W+Q_{l j}\right) \\
= & \sum_{l=1}^{n}\left(P_{l i} P_{l j}\right)\left(\Delta_{l i} W \Delta_{l j} W\right)+\sum_{l=1}^{n}\left(P_{l i} Q_{l j}\right) \Delta_{l i} W \\
& +\sum_{l=1}^{n}\left(P_{l j} Q_{l i}\right) \Delta_{l j} W+\sum_{l=1}^{n} Q_{l i} Q_{l j} .
\end{aligned}
$$

By Hölder's inequality,

$$
\sum_{l=1}^{n} Q_{l i}^{2} \leq \sum_{l=1}^{n} \int_{t_{l-1}+s_{i}}^{t_{l}+s_{i}} \int_{t_{l-1}+s_{i}}^{t_{l}+s_{i}}\left|D_{t} u_{i}(s)\right|^{2} d s d t
$$

Thus $\lim _{n \rightarrow \infty} E \sum_{l=1}^{n} Q_{l i}^{2}=0$. Now

$$
\begin{aligned}
\sum_{l=1}^{n}\left(P_{l i} \Delta_{l i} W\right)^{2} & =\sum_{l=1}^{n} \frac{\left(\Delta_{l i} W\right)^{2}}{\left(t_{l}-t_{l-1}\right)^{2}}\left(\int_{t_{l-1}+s_{i}}^{t_{l}+s_{i}} u_{i}(s) d s\right)^{2} \\
& =\sum_{l=1}^{n} \frac{\left(\Delta_{l i} W\right)^{2}}{t_{l}-t_{l-1}} \int_{t_{l-1}+s_{i}}^{t_{l}+s_{i}}\left(u_{i}^{n}(s)\right)^{2} d s
\end{aligned}
$$

It is easy to check that $E\left\|\left(u_{i}^{n}\right)^{2}\right\|_{L^{2}\left(\left[0, a+s_{i}\right]\right)} \leq E\left\|u_{i}^{2}\right\|_{L^{2}\left(\left[0, a+s_{i}\right]\right)}$ and

$$
\lim _{n \rightarrow \infty} E\left\|\left(u_{i}^{n}\right)^{2}-u_{i}^{2}\right\|_{L^{2}\left(\left[0, a+s_{i}\right]\right)}=0 .
$$

By an argument similar to the one used in the proof of Lemma A.2, we can show that $\left\{\sum_{l=1}^{n}\left(P_{l i} \Delta_{l i} W\right)^{2}, n \geq 1\right\}$ is uniformly integrable. Applying Lemma A.2, we have

$$
\lim _{n \rightarrow \infty} E\left|\sum_{l=1}^{n}\left(P_{l i} \Delta_{l i} W\right)^{2}-\int_{0}^{a+s_{i}} u_{i}^{2}(s) d s\right|=0 .
$$

The Cauchy-Schwarz-type inequality

$$
E\left|\sum_{l=1}^{n}\left(P_{l i} \Delta_{l i} W\right) Q_{l i}\right| \leq \sqrt{E \sum_{l=1}^{n}\left(P_{l i} \Delta_{l i} W\right)^{2} E \sum_{l=1}^{n} Q_{l i}^{2}}
$$

together with (2.19) and (2.21) implies that $\lim _{n \rightarrow \infty} E\left|\sum_{l=1}^{n}\left(P_{l i} \Delta_{l i} W\right) Q_{l i}\right|=0$.

Now consider the case $i \neq j$. The Cauchy-Schwarz inequality implies

$$
E\left|\sum_{l=1}^{n} Q_{l j} Q_{l i}\right| \leq \sqrt{E \sum_{l=1}^{n} Q_{l j}^{2} E \sum_{l=1}^{n} Q_{l i}^{2}} .
$$


We may write

$$
\begin{aligned}
\sum_{l=1}^{n}( & \left.P_{l i} P_{l j}\right)\left(\Delta_{l i} W \Delta_{l j} W\right) \\
& =\sum_{l=1}^{n} \frac{\Delta_{l i} W \Delta_{l j} W}{\left(t_{l}-t_{l-1}\right)^{2}} \int_{t_{l-1}+s_{i}}^{t_{l}+s_{i}} u_{i}(s) d s \int_{t_{l-1}+s_{j}}^{t_{l}+s_{j}} u_{j}(s) d s \\
& =\sum_{l=1}^{n} \frac{\Delta_{l i} W \Delta_{l j} W}{t_{l}-t_{l-1}} \int_{t_{l-1}+s_{i}}^{t_{l}+s_{i}} u_{i}^{n}(s) \hat{u}_{j}^{n}(s) d s
\end{aligned}
$$

where

$$
\hat{u}_{j}^{n}(s)=\sum_{l=1}^{m} \frac{I_{\left(t_{l-1}+s_{i}, t_{l}+s_{i}\right]}(s)}{t_{l}-t_{l-1}} \int_{t_{l-1}+s_{i}}^{t_{l}+s_{i}} u_{j}\left(s^{\prime}+s_{j}-s_{i}\right) d s^{\prime} .
$$

Similar to the case $i=j$, we have

$$
\lim _{n \rightarrow \infty} E\left|\sum_{l=1}^{n}\left(P_{l i} P_{l j}\right)\left(\Delta_{l i} W \Delta_{l j} W\right)\right|=0
$$

This completes the proof of the proposition.

Suppose that

$$
\bar{X}_{t_{l}}=X_{t_{l-1}}+\alpha_{l}\left(X_{t_{l}}-X_{t_{l-1}}\right)
$$

for some random variables $0 \leq \alpha_{l} \leq 1, l=1, \ldots, n$. Denote

$$
\begin{aligned}
\Delta\left(\Pi\left(X_{t_{l}}\right)\right) & =\left(\Pi\left(\Delta X_{t_{l}}\right)\right)=\Pi\left(X_{t_{l}}\right)-\Pi\left(X_{t_{l-1}}\right), \\
\Pi\left(\bar{X}_{t_{l}}\right) & =\Pi\left(X_{t_{l-1}}\right)+\alpha_{l} \Delta \Pi\left(X_{t_{l}}\right), \\
\Delta_{l i} X & =X\left(t_{l}+s_{i}\right)-X\left(t_{l-1}+s_{i}\right), \quad \text { for } 1 \leq i \leq k \text { and } 1 \leq l \leq n .
\end{aligned}
$$

Proposition 2.3. Suppose that $\phi \in C^{1,2}\left(T \times \mathbf{R}^{k}, \mathbf{R}\right)$, and let $1 \leq i, j \leq k$. Under the hypotheses of Proposition 2.2, we have

$$
\begin{aligned}
& \sum_{l=1}^{n} \frac{\partial^{2} \phi}{\partial x_{i} \partial x_{j}}\left(t_{l-1}, \Pi\left(\bar{X}_{t_{l}}\right)\right) \Delta_{l i} X \Delta_{l j} X \\
& \rightarrow \begin{cases}\int_{0}^{t+s_{i}} \frac{\partial^{2} \phi}{\partial x_{i}^{2}}\left(s, \Pi\left(X_{s}\right)\right) u^{2}(s) d s, & i=j, \\
0, & i \neq j\end{cases}
\end{aligned}
$$

as $n \rightarrow \infty$, in probability. 
PROOF. For $1 \leq i, j \leq k$,

$$
\begin{aligned}
\Delta_{l i} X \Delta_{l j} X= & \left(\Delta_{l i} U+\Delta_{l i} V\right)\left(\Delta_{l j} U+\Delta_{l j} V\right) \\
= & \Delta_{l i} U \Delta_{l j} U+\Delta_{l i} U \Delta_{l j} V \\
& +\Delta_{l i} V \Delta_{l j} U+\Delta_{l i} V \Delta_{l j} V,
\end{aligned}
$$

where $U, V$ are defined by (2.2). Since $U, V$ are continuous and $V \mid[0, a]$ is of bounded variation, it follows that

$$
\begin{aligned}
& \lim _{n \rightarrow \infty} \sum_{l=1}^{n} \Delta_{l i} U \Delta_{l j} V=0, \\
& \lim _{n \rightarrow \infty} \sum_{l=1}^{n} \Delta_{l i} V \Delta_{l j} U=0, \\
& \lim _{n \rightarrow \infty} \sum_{l=1}^{n} \Delta_{l i} V \Delta_{l j} V=0,
\end{aligned}
$$

in probability, for all $0 \leq i, j \leq n$. To handle the term $\sum_{l=1}^{n} \Delta_{l i} U \Delta_{l j} U$, we adapt an approach by Nualart and Pardoux (cf. [23], Theorem 3.4, or [22], Theorem 3.2.1).

Set

$$
Y(s):=\frac{\partial^{2} \phi}{\partial x_{i}^{2}}\left(s, \Pi\left(X_{s}\right)\right) I_{[0, t]}(s)
$$

and

$$
Y^{n}(s):=Y(0) I_{\{0\}}(s)+\sum_{l=1}^{n} \frac{\partial^{2} \phi}{\partial x_{i}^{2}}\left(t_{l-1}, \Pi\left(\bar{X}_{t_{l}}\right)\right) I_{\left(t_{l-1}, t_{l}\right]}(s) .
$$

Then $Y^{n}(s) \rightarrow Y(s)$ as $n \rightarrow \infty$, uniformly in $s \in[0, t]$. Applying Proposition 2.2 and Lemma A.3, we get

$$
\begin{aligned}
& \sum_{l=1}^{n} \frac{\partial^{2} \phi}{\partial x_{i} \partial x_{j}}\left(t_{l-1}, \Pi\left(\bar{X}_{t_{l}}\right)\right) \Delta_{l i} X \Delta_{l j} X \\
& \quad \rightarrow \delta_{i j} \int_{0}^{t+s_{i}} \frac{\partial^{2} \phi}{\partial x_{i}^{2}}\left(s, \Pi\left(X_{s}\right)\right) u^{2}(s) d s
\end{aligned}
$$

in probability as $n \rightarrow \infty$.

Proposition 2.4. Suppose that $\phi \in C^{1,2}\left(T \times \mathbf{R}^{k}\right)$ and let $X(t)$ be a continuous stochastic process defined by (2.1), where $u \in \mathbb{L}_{\mathrm{loc}}^{2,4}, v \in \mathbb{L}_{\mathrm{loc}}^{1,4}$ and $\eta \in$ $C\left([-\tau, 0], \mathbf{R}^{m}\right)$ is of bounded variation. Assume that $\pi_{n}:-\tau=s_{0}<\cdots<s_{n}=0$ 
are partitions of $[-\tau, 0]$ such that $\left|\pi_{n}\right| \rightarrow 0$ as $n \rightarrow \infty$. Then, for each $1 \leq i \leq k$ and each $t \in T$, we have

$$
\begin{aligned}
\lim _{n \rightarrow \infty} \sum_{l=1}^{n} \frac{\partial \phi}{\partial x_{i}}\left(t_{l-1}, \Pi\left(X_{t_{l-1}}\right)\right) \Delta_{l i} X \\
=\int_{0}^{t} \frac{\partial \phi}{\partial x_{i}}\left(s, \Pi\left(X_{s}\right)\right) d X\left(s+s_{i}\right) \\
\quad+\sum_{j=i+1}^{k} \int_{0}^{t} \frac{\partial^{2} \phi}{\partial x_{i} \partial x_{j}}\left(s, \Pi\left(X_{s}\right)\right) u^{2}\left(s+s_{i}\right) d s \\
+\sum_{j=1}^{k} \int_{0}^{t} \frac{\partial^{2} \phi}{\partial x_{i} \partial x_{j}}\left(s, \Pi\left(X_{s}\right)\right) \\
\times\left[\int_{0}^{s+s_{j}} D_{s+s_{i}} u(r) d W(r)\right. \\
\left.\quad+\int_{0}^{s+s_{j}} D_{s+s_{i}} v(r) d r\right] u\left(s+s_{i}\right) d s
\end{aligned}
$$

in probability.

PROOF. By a localization argument, we may assume that $\phi \in C_{b}^{1,2}(T \times$ $\left.\mathbf{R}^{k}, \mathbf{R}\right)$. Let $\left|\pi_{n}\right|<\min _{\{1 \leq i \leq k\}}\left|s_{i}-s_{i-1}\right|$. Fix $1 \leq i \leq k, 1 \leq l \leq n$, and set

$$
F_{l}:=\frac{\partial \phi}{\partial x_{i}}\left(t_{l-1}, \Pi\left(X_{t_{l-1}}\right)\right) \text {. }
$$

By property of the Skorohod integral ([23], Theorem 3.2), it follows that

$$
F_{l} \Delta_{l i} U=\int_{t_{l-1}+s_{i}}^{t_{l}+s_{i}} u(s) F_{l} d W(s)+\int_{t_{l-1}+s_{i}}^{t_{l}+s_{i}} D_{r}\left(F_{l}\right) u(r) d r,
$$

where $U$ is defined by (2.2). The chain rule (for weak derivatives) yields

$$
D_{r}\left(F_{l}\right)=\sum_{j=1}^{k} \frac{\partial^{2} \phi}{\partial x_{i} \partial x_{j}}\left(t_{l-1}, \Pi\left(X_{t_{l-1}}\right)\right) D_{r} X\left(t_{l-1}+s_{j}\right) .
$$

Now, taking the Malliavin derivative $D_{r}$ in (2.1) gives

$$
D_{r} X(t)=u(r) I_{\{r \leq t\}}+\int_{0}^{t} D_{r} u(s) d W(s)+\int_{0}^{t} D_{r} v(s) d s .
$$

Consequently,

$$
\sum_{l=1}^{n} \frac{\partial \phi}{\partial x_{i}}\left(t_{l-1}, \Pi\left(X_{t_{l-1}}\right)\right) \Delta_{l i} U=c_{1}+c_{2}+c_{3}+c_{4},
$$


where

$$
\begin{aligned}
& c_{1}:= \sum_{l=1}^{n} \int_{t_{l-1}+s_{i}}^{t_{l}+s_{i}} \frac{\partial \phi}{\partial x_{i}}\left(t_{l-1}, \Pi\left(X_{t_{l-1}}\right)\right) u(s) d W(s), \\
& c_{2}:= \sum_{l=1}^{n} \int_{t_{l-1}+s_{i}}^{t_{l}+s_{i}} \sum_{j=1}^{k} \frac{\partial^{2} \phi}{\partial x_{i} \partial x_{j}}\left(t_{l-1}, \Pi\left(X_{t_{l-1}}\right)\right) I_{\left\{r \leq t_{l-1}+s_{j}\right\}} u^{2}(r) d r, \\
& c_{3}:=\sum_{l=1}^{n} \int_{t_{l-1}+s_{i}}^{t_{l}+s_{i}} \sum_{j=1}^{k} \frac{\partial^{2} \phi}{\partial x_{i} \partial x_{j}}\left(t_{l-1}, \Pi\left(X_{t_{l-1}}\right)\right) \\
& \quad \times \int_{0}^{t_{l-1}+s_{j}} D_{r} u(s) d W(s) u(r) d r, \\
& c_{4}:=\sum_{l=1}^{n} \int_{t_{l-1}+s_{i}}^{t_{l}+s_{i}} \sum_{j=1}^{k} \frac{\partial^{2} \phi}{\partial x_{i} \partial x_{j}}\left(t_{l-1}, \Pi\left(X_{t_{l-1}}\right)\right) \\
& \times \int_{0}^{t_{l-1}+s_{j}} D_{r} v(s) d s u(r) d r .
\end{aligned}
$$

We will study the limits of the above expressions as $n \rightarrow \infty$.

Step 1. First we show that the limit of $c_{2}$ is given by

$$
c_{2} \rightarrow \sum_{j=i+1}^{k} \int_{0}^{t+s_{i}} \frac{\partial^{2} \phi}{\partial x_{i} \partial x_{j}}\left(r-s_{i}, \Pi\left(X_{r-s_{i}}\right)\right) u^{2}(r) d r \quad \text { a.s. }
$$

If $j \leq i$, then $t_{l-1}+s_{i} \geq t_{l-1}+s_{j}$. So when $t_{l-1}+s_{i}<r<t_{l}+s_{i}$, $I_{\left\{r \leq t_{l-1}+s_{j}\right\}}=0$. We have

$$
\begin{aligned}
c_{2} & =\sum_{j=i+1}^{k} \sum_{l=1}^{n} \int_{t_{l-1}+s_{i}}^{t_{l}+s_{i}} \frac{\partial^{2} \phi}{\partial x_{i} \partial x_{j}}\left(t_{l-1}, \Pi\left(X_{t_{l-1}}\right)\right) I_{\left\{r \leq t_{l-1}+s_{j}\right\}} u^{2}(r) d r \\
& \rightarrow \sum_{j=i+1}^{k} \int_{0}^{t+s_{i}} \frac{\partial^{2} \phi}{\partial x_{i} \partial x_{j}}\left(r-s_{i}, \Pi\left(X_{r-s_{i}}\right)\right) u^{2}(r) d r
\end{aligned}
$$

a.s. as $n \rightarrow \infty$.

Step 2. Next we study the limit of $c_{3}$ as $n \rightarrow \infty$. We claim that

$$
\begin{aligned}
c_{3} \rightarrow \sum_{j=1}^{k} \int_{0}^{t+s_{i}} & \frac{\partial^{2} \phi}{\partial x_{i} \partial x_{j}}\left(r-s_{i}, \Pi\left(X_{r-s_{i}}\right)\right) \\
& \times \int_{0}^{r-s_{i}+s_{j}} D_{r} u(s) d W(s) u(r) d r
\end{aligned}
$$


as $k \rightarrow \infty$ in probability. In fact,

$$
\begin{aligned}
& T_{j}^{n}:=\mid \sum_{l=1}^{n} \int_{t_{l-1}+s_{i}}^{t_{l}+s_{i}}\left[\frac{\partial^{2} \phi}{\partial x_{i} \partial x_{j}}\left(t_{l-1}, \Pi\left(X_{t_{l-1}}\right)\right) \int_{0}^{t_{l-1}+s_{j}} D_{r} u(s) d W(s)\right. \\
& -\int_{0}^{t+s_{i}} \frac{\partial^{2} \phi}{\partial x_{i} \partial x_{j}}\left(r-s_{i}, \Pi\left(X_{r-s_{i}}\right)\right) \\
& \left.\times \int_{0}^{r-s_{i}+s_{j}} D_{r} u(s) d W(s)\right] u(r) d r \\
& \leq\left|\sum_{l=1}^{n} \int_{t_{l-1}+s_{i}}^{t_{l}+s_{i}} \frac{\partial^{2} \phi}{\partial x_{i} \partial x_{j}}\left(t_{l-1}, \Pi\left(X_{t_{l-1}}\right)\right) \int_{t_{l-1}+s_{j}}^{r+s_{j}-s_{i}} D_{r} u(s) d W(s) u(r) d r\right| \\
& +\mid \sum_{l=1}^{n} \int_{t_{l-1}+s_{i}}^{t_{l}+s_{i}}\left[\frac{\partial^{2} \phi}{\partial x_{i} \partial x_{j}}\left(t_{l-1}, \Pi\left(X_{t_{l-1}}\right)\right)-\frac{\partial^{2} \phi}{\partial x_{i} \partial x_{j}}\left(r-s_{i}, \Pi\left(X_{r-s_{i}}\right)\right)\right] \\
& \times \int_{0}^{r-s_{i}+s_{j}} D_{r} u(s) d W(s) u(r) d r \\
& \leq\left\|\frac{\partial^{2} \phi}{\partial x_{i} \partial x_{j}}\right\|_{\infty} \sum_{l=1}^{n} \int_{t_{l-1}+s_{i}}^{t_{l}+s_{i}}\left|\int_{t_{l-1}+s_{j}}^{r+s_{j}-s_{i}} D_{r} u(s) d W(s)\right||u(r)| d r \\
& +\sup _{1 \leq l \leq n} \sup _{r \in\left[t_{l-1}+s_{i}, t_{l}+s_{i}\right]} \mid \frac{\partial^{2} \phi}{\partial x_{i} \partial x_{j}}\left(t_{l-1}, \Pi\left(X_{t_{l-1}}\right)\right) \\
& -\frac{\partial^{2} \phi}{\partial x_{i} \partial x_{j}}\left(r-s_{i}, \Pi\left(X_{r-s_{i}}\right)\right) \\
& \times \int_{0}^{t_{l}+s_{i}}\left|\int_{0}^{r-s_{i}+s_{j}} D_{r} u(s) d W(s) u(r)\right| d r \\
& =T_{j 1}^{n}+T_{j 2}^{n},
\end{aligned}
$$

where $T_{j 1}^{n}$ and $T_{j 2}^{n}$ denote the first and second term on the right-hand side of the last inequality. Using the Cauchy-Schwarz inequality and the $L^{p}$ inequality for the Skorohod integral ([23], Proposition 3.5, and [22], page 158), we have

$$
\begin{aligned}
E T_{j 1}^{n} \leq & \left\|\frac{\partial^{2} \phi}{\partial x_{i} \partial x_{j}}\right\|_{\infty}\left(E \int_{0}^{a+s_{i}} u^{2}(r) d r\right)^{1 / 2} \\
& \times\left\{E \sum_{l=1}^{n} \int_{t_{l-1}+s_{i}}^{t_{l}+s_{i}} \int_{t_{l-1}+s_{j}}^{r+s_{j}-s_{i}}\left|D_{r} u(s)\right|^{2} d s d r\right. \\
& \left.\quad+E \sum_{l=1}^{n} \int_{t_{l-1}+s_{i}}^{t_{l}+s_{i}} \int_{t_{l-1}+s_{j}}^{r+s_{j}-s_{i}} \int_{0}^{a}\left|D_{\theta}\left(D_{r} u(s)\right)\right|^{2} d \theta d s d r\right\}^{1 / 2} \rightarrow 0
\end{aligned}
$$


as $n \rightarrow \infty$. The uniform continuity of $\frac{\partial^{2} \phi}{\partial x_{i} \partial x_{j}}$ implies $T_{j 2}^{n} \rightarrow 0$ a.s. So as $n \rightarrow \infty$, $T_{j}^{n} \rightarrow 0$ in probability.

Step 3. Now we will show that

$$
\begin{aligned}
c_{4} \rightarrow \sum_{j=1}^{k} \int_{0}^{t+s_{i}} & \frac{\partial^{2} \phi}{\partial x_{i} \partial x_{j}}\left(r-s_{i}, \Pi\left(X_{r-s_{i}}\right)\right) \\
& \times \int_{0}^{r+s_{j}-s_{i}} D_{r} v(s) d s u(r) d r \quad \text { a.s. }
\end{aligned}
$$

As in Step 2, we have

$$
\begin{aligned}
& \mid \sum_{l=1}^{n} \int_{t_{l-1}+s_{i}}^{t_{l}+s_{i}}\left[\frac{\partial^{2} \phi}{\partial x_{i} \partial x_{j}}\left(t_{l-1}, \Pi\left(X_{t_{l-1}}\right)\right) \int_{0}^{t_{l-1}+s_{j}} D_{r} v(s) d s\right. \\
& \left.\quad-\frac{\partial^{2} \phi}{\partial x_{i} \partial x_{j}}\left(r-s_{i}, \Pi\left(X_{r-s_{i}}\right)\right) \int_{0}^{r-s_{i}+s_{j}} D_{r} v(s) d s\right] u(r) d r \mid \\
& \leq \| \frac{\partial^{2} \phi}{\partial x_{i} \partial x_{j}}\left|\sum_{\infty} \sum_{l=1}^{n} \int_{t_{l-1}+s_{i}}^{t_{l}+s_{i}}\right| \int_{t_{l-1}+s_{j}}^{r+s_{j}-s_{i}} D_{r} v(s) d s|| u(r) \mid d r \\
& +\sup _{1 \leq l \leq n} \mid \frac{\partial^{2} \phi}{\partial x_{i} \partial x_{j}}\left(t_{l-1}, \Pi\left(X_{t_{l-1}}\right)\right) \\
& \quad-\frac{\partial^{2} \phi}{\partial x_{i} \partial x_{j}}\left(r-s_{i}, \Pi\left(X_{r-s_{i}}\right)\right) \mid \\
& \quad \times \int_{0}^{t_{l}+s_{i}}\left|\int_{0}^{r-s_{i}+s_{j}} D_{r} v(s) d s\right||u(r)| d r \\
& \rightarrow 0 \quad \text { a.s. as } n \rightarrow \infty .
\end{aligned}
$$

Step 4. Finally, we study the limit of $c_{1}$ as $n \rightarrow \infty$. We shall show that

$$
c_{1} \rightarrow \int_{0}^{t+s_{i}} \frac{\partial \phi}{\partial x_{i}}\left(s-s_{i}, \Pi\left(X_{s-s_{i}}\right)\right) u(s) d W(s)
$$

in $L^{2}(\Omega, \mathbf{R})$ as $n \rightarrow \infty$. To see this, define

$$
u^{n}(s):=u(s) \sum_{l=1}^{n} \frac{\partial \phi}{\partial x_{i}}\left(t_{l-1}, \Pi\left(X_{t_{l-1}}\right)\right) I_{\left(t_{l-1}+s_{i}, t_{l}+s_{i}\right]}(s) .
$$

It suffices to show that

$$
u^{n}(s) \rightarrow \frac{\partial \phi}{\partial x_{i}}\left(s-s_{i}, \Pi\left(X_{t_{s-s_{i}}}\right)\right) u(s) I_{\left(0, t+s_{i}\right]}(s)
$$


in $\mathbb{L}^{1,2}$ as $n \rightarrow \infty$. It is clear that the sequence $\left\{u^{n}(s)\right\}$ converges to $\frac{\partial \phi}{\partial x_{i}}\left(s-s_{i}\right.$, $\left.\Pi\left(X_{s-s_{i}}\right)\right) u(s) I_{\left(0, t+s_{i}\right]}(s)$ in $L^{2}(\Omega \times T, \mathbf{R})$. It remains to show that the sequence $\left\{D_{r} u^{n}(s)\right\}_{n=1}^{\infty}, r, s \in T$, converges in $L^{2}\left(\Omega \times T^{2}, \mathbf{R}\right)$ to $D_{r}\left[\frac{\partial \phi}{\partial x_{i}}\left(s-s_{i}\right.\right.$, $\left.\left.\Pi\left(X_{s-s_{i}}\right)\right) u(s) I_{\left(0, t+s_{i}\right]}(s)\right]$. Now

$$
\begin{aligned}
& D_{r} u^{n}(s)= D_{r} u(s) \sum_{l=1}^{n} \frac{\partial \phi}{\partial x_{i}}\left(t_{l-1}, \Pi\left(X_{t_{l-1}}\right)\right) I_{\left(t_{l-1}+s_{i}, t_{l}+s_{i}\right]}(s) \\
&+u(s) \sum_{l=1}^{n}\left[\sum_{j=1}^{k} \frac{\partial^{2} \phi}{\partial x_{i} \partial x_{j}}\left(t_{l-1}, \Pi\left(X_{t_{l-1}}\right)\right) \int_{0}^{t_{l-1}+s_{j}} D_{r} u\left(s^{\prime}\right) d W\left(s^{\prime}\right)\right] \\
& \times I_{\left.t_{l-1}+s_{i}, t_{l}+s_{i}\right]}(s) \\
&+u(s) \sum_{l=1}^{n}\left[\sum_{j=1}^{k} \frac{\partial^{2} \phi}{\partial x_{i} \partial x_{j}}\left(t_{l-1}, \Pi\left(X_{t_{l-1}}\right)\right) \int_{0}^{t_{l-1}+s_{j}} D_{r} v\left(s^{\prime}\right) d s^{\prime}\right] \\
& \times I_{\left.I_{l-1}+s_{i}, t_{l}+s_{i}\right]}(s) \\
&+u(s) \sum_{l=1}^{n}\left[\sum_{j=1}^{k} \frac{\partial^{2} \phi}{\partial x_{i} \partial x_{j}}\left(t_{l-1}, \Pi\left(X_{t_{l-1}}\right)\right) u(r) I_{\left[0, t_{l-1}+s_{j}\right]}(r)\right] \\
& \times I_{\left.t_{l-1}+s_{i}, t_{l}+s_{i}\right]}(s) \\
&=d_{1}+d_{2}+d_{3}+d_{4},
\end{aligned}
$$

where $d_{1}, d_{2}, d_{3}$ and $d_{4}$ stand for the first, second, third and fourth terms on the right-hand side of the above equality, respectively. It is easy to see that

$$
d_{1} \rightarrow D_{r} u(s) \frac{\partial \phi}{\partial x_{i}}\left(\Pi\left(s-s_{i}, X_{s-s_{i}}\right)\right) I_{\left(0, t+s_{i}\right]}(s)
$$

in $L^{2}(\Omega, \mathbf{R})$. Since for all $1 \leq j \leq k, u(s) \int_{0}^{s+s_{j}-s_{i}} D_{r} v(\theta) d \theta$ belongs to $L^{2}(\Omega \times$ $\left.T^{2}, \mathbf{R}\right)$, then by Lebesgue's dominated convergence theorem, the $L^{2}\left(\Omega \times T^{2}, \mathbf{R}\right)$ limit of the function $q_{3}(s, r)$ defined by

$$
q_{3}:=u(s) \sum_{j=1}^{k} \sum_{l=1}^{n}\left[\frac{\partial^{2} \phi}{\partial x_{i} \partial x_{j}}\left(t_{l-1}, \Pi\left(X_{t_{l-1}}\right)\right) \int_{0}^{s+s_{j}-s_{i}} D_{r} v(\theta) d \theta\right] I_{\left(t_{l-1}+s_{i}, t_{l}+s_{i}\right]}(s)
$$

is

$$
\sum_{j=1}^{k} u(s)\left[\frac{\partial^{2} \phi}{\partial x_{i} \partial x_{j}}\left(s-s_{i}, \Pi\left(X_{s-s_{i}}\right)\right) \int_{0}^{s+s_{j}-s_{i}} D_{r} v(\theta) d \theta\right] I_{\left(0, t+s_{i}\right]}(s) .
$$

Since $v \in \mathbb{L}^{1,4}$ and $u \in L^{4}(\Omega \times T, \mathbf{R})$, the following argument shows that the 
difference between $d_{3}$ and $q_{3}$ converges to 0 as $n \rightarrow \infty$ in $L^{1}(\Omega, \mathbf{R})$ :

$$
\begin{aligned}
& \sum_{l=1}^{n} \int_{t_{l-1}+s_{i}}^{t_{l}+s_{i}} \int_{0}^{a} u^{2}(s)\left[\frac{\partial^{2} \phi}{\partial x_{i} \partial x_{j}}\left(t_{l-1}, \Pi\left(X_{t_{l-1}}\right)\right)\right]^{2}\left[\int_{t_{l-1}+s_{j}}^{s+s_{j}-s_{i}} D_{r} v(\theta) d \theta\right]^{2} d r d s \\
& \quad \leq\left|\pi_{n}\right|\left\|\frac{\partial^{2} \phi}{\partial x_{i} \partial x_{j}}\right\|_{\infty}^{2} \int_{0}^{a} u^{2}(s) d s \int_{0}^{a} \int_{0}^{a}\left(D_{r} v(\theta)\right)^{2} d r d \theta \\
& \quad \rightarrow 0 .
\end{aligned}
$$

Hence, the $L^{2}\left(\Omega \times T^{2}, \mathbf{R}\right)$ limit of $d_{3}$ is the same as that of $q_{3}$, namely,

$$
\sum_{j=1}^{k} u(s)\left[\frac{\partial^{2} \phi}{\partial x_{i} \partial x_{j}}\left(s-s_{i}, \Pi\left(X_{s-s_{i}}\right)\right) \int_{0}^{s+s_{j}-s_{i}} D_{r} v(\theta) d \theta\right] I_{\left(0, t+s_{i}\right]}(s)
$$

in $L^{2}\left(\Omega \times T^{2}, \mathbf{R}\right)$. To find the limit of $d_{2}$, we need to check that for all $j$, the two-parameter process $\left(u(s) \int_{0}^{s+s_{j}-s_{i}} D_{r} u(\theta) d W(\theta), 0 \leq s, r \leq a\right)$ belongs to $L^{2}\left(\Omega \times T^{2}, \mathbf{R}\right)$. This follows from the following estimates:

$$
\begin{aligned}
E \int_{0}^{a} \int_{0}^{a} u^{2}(s)\left[\int_{0}^{s+s_{j}-s_{i}} D_{r} u(\theta) d W(\theta)\right]^{2} d s d r \\
\leq\left\{E \int_{0}^{a} u^{4}(s) d s E \int_{0}^{a}\left(\int_{0}^{a}\left[\int_{0}^{s+s_{j}-s_{i}} D_{r} u(\theta) d W(\theta)\right]^{2} d r\right)^{2} d s\right\}^{1 / 2} \\
\leq C\left\{E \int _ { 0 } ^ { a } u ^ { 4 } ( s ) d s \left[E\left(\int_{0}^{a} \int_{0}^{a}\left|D_{r} u(\theta)\right|^{2} d \theta d r\right)^{2}\right.\right. \\
\left.\left.+E\left(\int_{0}^{a} \int_{0}^{a} \int_{0}^{a} D_{\alpha}\left(D_{r} u(\theta)\right) d \theta d r d \alpha\right)^{2}\right]\right\}^{1 / 2} .
\end{aligned}
$$

Here we have used a slight modification of the $L^{p}$ estimate of the Skorohod integral for $p=4$ (cf. [23], Exercise 3.2.7). Using similar $L^{p}$ estimates to the above, we obtain

$$
\begin{aligned}
& \sum_{l=1}^{n} \int_{t_{l-1}+s_{i}}^{t_{l}+s_{i}} \int_{0}^{a} u^{2}(s)\left[\frac{\partial^{2} \phi}{\partial x_{i} \partial x_{j}}\left(t_{l-1}, \Pi\left(X_{t_{l-1}}\right)\right)\right]^{2} \\
& \times\left[\int_{t_{l-1}+s_{j}}^{s+s_{j}-s_{i}} D_{r} u(\theta) d W(\theta)\right]^{2} d r d s \\
& \leq\left\|\frac{\partial^{2} \phi}{\partial x_{i} \partial x_{j}}\right\|_{\infty}^{2}\left(\int_{0}^{a} E u^{4}(s) d s\right)^{1 / 2} \\
& \times\left\{\sum_{l=1}^{n} E \int_{t_{l-1}+s_{i}}^{t_{l}+s_{i}}\left[\int_{0}^{a}\left(\int_{t_{l-1}+s_{j}}^{s+s_{j}-s_{i}} D_{r} v(\theta) d \theta\right)^{2} d r\right]^{2} d s\right\}^{1 / 2} .
\end{aligned}
$$


Note that the right-hand side of the above inequality tends to zero as $n \rightarrow \infty$. Thus

$$
\begin{aligned}
d_{2} \rightarrow \sum_{j=1}^{k} u(s)[ & \frac{\partial^{2} \phi}{\partial x_{i} \partial x_{j}}\left(s-s_{i}, \Pi\left(X_{s-s_{i}}\right)\right) \\
& \left.\times \int_{0}^{s+s_{j}-s_{i}} D_{r} u(\theta) d W(\theta)\right] I_{\left(0, t+s_{i}\right]}(s)
\end{aligned}
$$

in $L^{2}\left(\Omega \times T^{2}, \mathbf{R}\right)$ as $n \rightarrow \infty$.

It is easy to check that

$$
d_{4} \rightarrow \sum_{j=1}^{k} u(s) \frac{\partial^{2} \phi}{\partial x_{i} \partial x_{j}}\left(s-s_{i}, \Pi\left(X_{s-s_{i}}\right)\right) u(r) I_{\left[0, s+s_{j}-s_{i}\right]}(r) I_{\left(0, t+s_{i}\right]}(s)
$$

as $n \rightarrow \infty$ in $L^{2}(\Omega, \mathbf{R})$. Therefore,

$$
D_{r} u^{n}(s) \rightarrow D_{r}\left[u(s) \frac{\partial^{2} \phi}{\partial x_{i} \partial x_{j}}\left(s-s_{i}, \Pi\left(X_{s-s_{i}}\right)\right) I_{\left(0, t+s_{i}\right]}(s)\right]
$$

in $L^{2}\left(\Omega \times T^{2}, \mathbf{R}\right)$. Finally it is easy to see that

$$
c_{1} \rightarrow \int_{0}^{t+s_{i}} \frac{\partial \phi}{\partial x_{i}}\left(s-s_{i}, \Pi\left(X_{s-s_{i}}\right)\right) u(s) d W(s)
$$

in $L^{2}(\Omega, \mathbf{R})$ as $n \rightarrow \infty$.

Step 5. The convergence

$$
\begin{aligned}
& \sum_{l=1}^{n} \frac{\partial \phi}{\partial x_{i}}\left(t_{l-1}, \Pi\left(X_{t_{l-1}}\right)\right) \Delta_{l i} V \\
& \quad \rightarrow \int_{s_{i}}^{t+s_{i}} \frac{\partial \phi}{\partial x_{i}}\left(s-s_{i}, \Pi\left(X_{s-s_{i}}\right)\right) d V(s) \quad \text { a.s. }
\end{aligned}
$$

as $n \rightarrow \infty$, is easy to verify.

We complete the section by giving a Stratonovich version of the Itô formula (2.7).

Suppose that $k \geq 1$ and $p \geq 2$. The set $\mathbb{L}_{d, C}^{k, p}$ (cf. [23], Definition 7.2, and [22], page 167) is the class of processes $u \in L_{d}^{k, p}$ such that the mappings $s \hookrightarrow$ $D_{s \wedge t} u(s \vee t)$ and $s \hookrightarrow D_{s \vee t} u(s \wedge t)$ are continuous in $L^{p}(\Omega)$, uniformly in $t \in T$, and $\sup _{s, t \in T} E\left(\left|D_{s} u(t)\right|^{p}\right)<\infty$. 
The space $\mathbb{L}_{d, C, \text { loc }}^{1,2}$ is the class of processes that are locally in $\mathbb{L}_{d, C}^{1,2}$. For any $u \in \mathbb{L}_{d, C}^{1,2}$, the following limits,

$$
\begin{aligned}
& D_{t}^{+} u(t)=\lim _{\varepsilon \downarrow 0} \sum_{i=1}^{d} D_{t}^{i} u^{i}(t+\varepsilon), \\
& D_{t}^{-} u(t)=\lim _{\varepsilon \downarrow 0} \sum_{i=1}^{d} D_{t}^{i} u^{i}(t-\varepsilon),
\end{aligned}
$$

exist in $L^{2}(\Omega)$ uniformly in $t$, we set $\nabla=D^{+}+D^{-}$, that is, $(\nabla u)(t)=D_{t}^{+} u(t)+$ $D_{t}^{-} u(t)$.

Consider the process

$$
X(t)= \begin{cases}\eta(0)+\int_{0}^{t} u(s) \circ d W(s)+\int_{0}^{t} v(s) d s, & t>0, \\ \eta(t), & -\tau \leq t \leq 0,\end{cases}
$$

where $\eta$ belongs to $C$ and is of bounded variation, $u=\left(u^{1}, \ldots, u^{m}\right)^{T}, u^{i} \in$ $\mathbb{L}_{d, C, \text { loc }}^{2,4},(\nabla u) \in \mathbb{L}_{\mathrm{loc}}^{1,4}, v=\left(v^{1}, \ldots, v^{m}\right)^{T}, v^{i} \in \mathbb{L}_{\mathrm{loc}}^{1,4}$, and the stochastic integral is a Stratonovich one. Assume also that the process $X$ is continuous.

Using the relationship between the Skorohod and Stratonovich integrals ([23], Theorem 7.3, and [22], Theorem 3.11) and Theorem 2.3, we can easily obtain the following Stratonovich version of Itô's formula for the segment process $X_{t}$ (cf. [28]).

COROLlary 2.5. Suppose that the process $X(t)$ is defined by (2.52), and let $\phi \in C^{1,2}\left(T \times \mathbf{R}^{m k}, \mathbf{R}\right)$. Then

$$
\begin{aligned}
\phi\left(t, \Pi\left(X_{t}\right)\right)-\phi\left(0, \Pi\left(X_{0}\right)\right) \\
=\int_{0}^{t} \frac{\partial \phi}{\partial s}\left(s, \Pi\left(X_{s}\right)\right) d s \\
\quad+\sum_{i=1}^{k} \int_{0}^{t} \frac{\partial \phi}{\partial \vec{x}^{i}}\left(s, \Pi\left(X_{s}\right)\right) u\left(s+s_{i}\right) \circ d W\left(s+s_{i}\right) \\
\quad+\sum_{i=1}^{k} \int_{0}^{t} \frac{\partial \phi}{\partial \vec{x}^{i}}\left(s, \Pi\left(X_{s}\right)\right) v\left(s+s_{i}\right) d s
\end{aligned}
$$

for all $t \in T$ a.s.

3. Weak differentiability of solutions of SDDEs. In this section, we will study the weak differentiability of the solution of the Itô SDDE (1.6). Bell and Mohammed [6] have applied the Malliavin calculus to study regularity of solutions of SDDEs with a single delay in the noise term. Their analysis relies on weak 
differentiability of the solution of the SDDE. In Section 5 of this article, the weak differentiability of the solution to the $\operatorname{SDDE}$ (1.6) together with the Itô formula (2.10) are used to develop higher-order numerical schemes for solving the SDDE. The next three results (Proposition 3.1, Lemma 3.2 and Proposition 3.3) are analogous to those in [22], Theorem 2.2.1, Lemma 2.2.2 and Theorem 2.2.2. Denote $\mathbb{D}_{m}^{k, \infty}:=\bigcap_{p \geq 2} \mathbb{D}_{m}^{k, p}$, for $k \in N$. Recall that $D_{r}^{l}, 1 \leq l \leq d$, stand for weak differentiation with respect to the $l$ th component of $W$.

Proposition 3.1 (cf. [22], Proposition 1.2.3). In the Itô SDDE (1.6), assume that $g \in C_{b}^{0,1}\left(T \times \mathbf{R}^{k_{1} m}, L\left(\mathbf{R}^{d}, \mathbf{R}^{m}\right)\right)$ and $h \in C_{b}^{0,1}\left(T \times \mathbf{R}^{k_{2} m}, \mathbf{R}^{m}\right)$. Let $X$ be the solution of (1.6). Then $X(t) \in \mathbb{D}_{m}^{1, \infty}$ for all $t \in T$, and

$$
\sup _{0 \leq r \leq a} E\left(\sup _{r \leq s \leq a}\left|D_{r} X(s)\right|^{p}\right)<\infty
$$

for all $p \geq 2$. Furthermore, the "partial" weak derivatives $D_{r}^{l} X^{j}(t)$ with respect to the lth coordinate of $W$ satisfy the following linear SDDEs a.s.:

$$
D_{r}^{l} X^{j}(t)=\left\{\begin{array}{rlrl}
g^{j l}(r, & \left.\Pi_{1}\left(X_{r}^{j}\right)\right) \\
& +\int_{r}^{t} \sum_{i=1}^{k_{1}} \frac{\partial g^{j l}}{\partial \vec{x}_{i}}\left(s, \Pi_{1}\left(X_{s}\right)\right) D_{r}^{l} X^{j}\left(s+s_{1, i}\right) d W^{l}(s) & \\
& +\int_{0}^{t} \sum_{i=1}^{k_{2}} \frac{\partial h^{j}}{\partial \vec{x}_{i}}\left(s, \Pi_{2}\left(X_{s}\right)\right) D_{r}^{l} X^{j}\left(s+s_{2, i}\right) d s, & t \geq r, \\
0, & t<r
\end{array}\right.
$$

for $l=1, \ldots, d, j=1, \ldots, m$. In (3.2), $g^{j l}$ is the $(j, l)$ entry of the $m \times d$ matrix $g$, and $h^{j}$ is the jth coordinate of $h$.

ProOF. For simplicity, we will only consider the one-dimensional case $d=$ $m=1$

$$
X^{n+1}(t)=\eta(0)+\int_{0}^{t} g\left(s, \Pi_{1}\left(X_{s}^{n}\right)\right) d W(s)+\int_{0}^{t} h\left(s, \Pi_{2}\left(X_{s}^{n}\right)\right) d s .
$$

It is easy to see that

$$
\begin{aligned}
& D_{r}\left(\int_{0}^{t} g\left(s, \Pi_{1}\left(X_{s}^{n}\right)\right) d W(s)\right) \\
& \quad=g\left(r, \Pi_{1}\left(X_{r}^{n}\right)\right)+\int_{r-s_{1, k_{1}}}^{t} D_{r}\left(g\left(s, \Pi_{1}\left(X_{s}^{n}\right)\right)\right) d W(s)
\end{aligned}
$$


and

$$
D_{r}\left(\int_{0}^{t} h\left(s, \Pi_{2}\left(X_{s}^{n}\right)\right) d s\right)=\int_{r-s_{2, k_{2}}}^{t} D_{r}\left(h\left(s, \Pi_{2}\left(X_{s}^{n}\right)\right)\right) d s .
$$

Since $g$ and $h$ have bounded space derivatives, it is easy to see that there is a positive constant $K$ such that

$$
\begin{gathered}
\left\|D_{r}\left(g\left(s, \Pi_{1}\left(X_{s}^{n}\right)\right)\right)\right\| \leq K \sup _{r \leq u \leq s}\left|D_{r} X^{n}(u)\right|, \\
\left|D_{r}\left(h\left(s, \Pi_{2}\left(X_{s}^{n}\right)\right)\right)\right| \leq K \sup _{r \leq u \leq s}\left|D_{r} X^{n}(u)\right|,
\end{gathered}
$$

almost surely. From the Burkholder-Davis-Gundy inequality and (3.3)-(3.6), it follows that $X^{n}(t) \in \mathbb{D}^{1, \infty}$ for all $t \in[0, a]$, and there are positive constants $C_{1}, C_{2}$ such that

$$
\begin{aligned}
& E\left(\sup _{r \leq u \leq t}\left|D_{r} X^{n+1}(u)\right|^{p}\right) \\
& \quad \leq C_{1}\left(1+E\left\|X_{r}^{n}\right\|_{C}^{p}\right)+C_{2} \int_{r}^{t} E\left(\sup _{r \leq u \leq s}\left|D_{r} X^{n}(u)\right|^{p}\right) d s .
\end{aligned}
$$

By induction on $n$, the above inequality implies that $E\left(\sup _{r \leq s \leq a}\left|D_{r} X^{n}(s)\right|^{p}\right)$ are uniformly bounded in $n$ for all $p \geq 2$. By [22], Proposition 1.5.5, it follows that $X(t) \in \mathbb{D}^{1, \infty}$ for all $t$. Applying the operator $D$ to (1.6) (and using [22], Proposition 1.2.3), we obtain the linear SDDE (3.2) for the weak derivative of $X(t)$. The estimate (3.1) follows from (3.2), Burkholder-Davis-Gundy's inequality and Gronwall's lemma.

The following lemma may be proved using similar ideas. Its proof is left to the reader.

LEMMA 3.2. Suppose that the real-valued process $\alpha=\{\alpha(r, t): t \in[r, a]\}$ is adapted and continuous. Assume that the processes $a(t)=\left(a_{1}(t), \ldots, a_{k_{1}}(t)\right) \in$ $\mathbf{R}^{k_{1}}$ and $b(t)=\left(b_{1}(t), \ldots, b_{k_{2}}(t)\right) \in \mathbf{R}^{k_{2}}$ are adapted, continuous and uniformly bounded. Furthermore, suppose that the random variables $\alpha(r, t), a(t)$ and $b(t)$ belong to $\mathbb{D}^{1, \infty}$ and satisfy the conditions

$$
\begin{array}{r}
\sup _{0 \leq r \leq a} E\left(\sup _{r \leq t \leq a}|\alpha(r, t)|^{p}\right)+\sup _{0 \leq r, s \leq a} E\left(\sup _{s \leq t \leq a}\left|D_{s} \alpha(r, t)\right|^{p}\right)<\infty, \\
\sup _{0 \leq s \leq a}\left\{E\left(\sup _{s \leq t \leq a}|a(t)|^{p}\right)+E\left(\sup _{s \leq t \leq a}\left|D_{s} a(t)\right|^{p}\right)\right\}<\infty, \\
\sup _{0 \leq s \leq a}\left\{E\left(\sup _{s \leq t \leq a}|b(t)|^{p}\right)+E\left(\sup _{s \leq t \leq a}\left|D_{s} b(t)\right|^{p}\right)\right\}<\infty
\end{array}
$$


for all $p \geq 2$. Let $Y=\{Y(t): t \in[0, a]\}$ be the solution of the linear SDDE

$$
Y(t)=\left\{\begin{array}{l}
\alpha(r, t)+\int_{r}^{t}\left\langle a(s), \Pi_{1}\left(Y_{s}\right)\right\rangle_{\mathbf{R}^{k_{1}}} d W(s)+\int_{r}^{t}\left\langle b(s), \Pi_{2}\left(Y_{s}\right)\right\rangle_{\mathbf{R}^{k_{2}}} d s, \\
0, \quad 0 \leq t \leq r .
\end{array}\right.
$$

Then $Y(t)$ belongs to $\mathbb{D}^{1, \infty}$, and for all integers $p \geq 2$, we have

$$
\begin{gathered}
\sup _{0 \leq s \leq a} E\left(\sup _{s \leq t \leq a}\left|D_{s} Y(t)\right|^{p}\right)<\infty \\
\sup _{0 \leq s \leq a} E\left(\sup _{s \leq t \leq a}|Y(t)|^{p}\right)<\infty .
\end{gathered}
$$

Furthermore, the weak derivative $D_{S} Y(t)$ of $Y(t)$ satisfies the linear SDDE

$$
D_{s} Y(t)=D_{s} \alpha(r, t)+\left\langle a(s), \Pi_{1}\left(Y_{s}\right)\right\rangle_{\mathbf{R}^{k_{1}}} I_{\{r \leq s \leq t\}}
$$

$$
\begin{aligned}
& +\int_{r}^{t}\left[\left\langle D_{s} a(v), \Pi_{1}\left(Y_{v}\right)\right\rangle_{\mathbf{R}^{k_{1}}}+\left\langle a(v), \Pi_{1}\left(D_{s} Y_{v}\right)\right\rangle_{\mathbf{R}^{k_{1}}}\right] d W(v) \\
& +\int_{r}^{t}\left[\left\langle D_{s} b(v), \Pi_{2}\left(Y_{v}\right)\right\rangle_{\mathbf{R}^{k_{2}}}+\left\langle b(v), \Pi_{2}\left(D_{s} Y_{v}\right)\right\rangle_{\mathbf{R}^{k_{2}}}\right] d v, \quad s<t .
\end{aligned}
$$

The next proposition follows from Proposition 3.1 and Lemma 3.2.

Proposition 3.3. Let $X=\{X(t): t \in T=[0, a]\}$ be the solution of the $\operatorname{SDDE}$ (1.6), where $g \in C_{b}^{0,2}\left(T \times \mathbf{R}^{k_{1} m}, L\left(\mathbf{R}^{d}, \mathbf{R}^{m}\right)\right), h \in C_{b}^{0,2}\left(T \times \mathbf{R}^{k_{2} m}, \mathbf{R}^{m}\right)$ have bounded first and second partial derivatives in the space variables. Then $X(t) \in \mathbb{D}_{m}^{2, \infty}$ for all $t \in T$, and

$$
\sup _{0 \leq r_{1}, r_{2} \leq a} E\left(\sup _{r_{1} \vee r_{2} \leq s \leq a}\left|D_{r_{1}}^{l_{1}} D_{r_{2}}^{l_{2}} X(s)\right|^{p}\right)<\infty
$$

for $l_{1}, l_{2}=1, \ldots, d$, and all $p \geq 2$.

4. Strong approximation of multiple Stratonovich integrals. The following iterated Stratonovich integrals are used in the Milstein scheme for the SDDE (1.6):

$$
J_{i, j}\left(t_{0}, t_{1} ;-b\right):=\int_{t_{0}+b}^{t_{1}+b} \int_{t_{0}}^{s-b} \circ d W^{i}(v) \circ d W^{j}(s),
$$

where $0<t_{0}<t_{1}, b \geq 0$.

We will adopt the discretization scheme in [17], Section 5.8, in order to handle the above double stochastic integral. For alternative discretization approaches to iterated stochastic integrals, see [11] and [26]. 
Set

$$
J\left(t_{0}, t_{1} ;-b\right):=J_{1,1}\left(t_{0}, t_{1} ;-b\right),
$$

$t:=t_{1}-t_{0}$ and $r:=2 \pi / t$. We choose a complete orthonormal basis of $L^{2}[0, t]$ as

$$
\left\{\frac{1}{\sqrt{t}}, \sqrt{\frac{2}{t}} \sin n r s, \sqrt{\frac{2}{t}} \cos n r s: n=1,2, \ldots, 0 \leq s \leq t\right\} .
$$

Set $\bar{W}^{i}(s):=W^{i}\left(s+t_{0}\right)-W^{i}\left(t_{0}\right)$ and $\bar{B}^{j}(s):=\bar{W}^{j}(s+b)-\bar{W}^{j}(b), s \geq 0$, $1 \leq i, j \leq d$. Using the Kahunen-Loève expansion technique, we have

$$
\bar{W}^{i}(s)-\frac{s}{t} \bar{W}^{i}(t)=\frac{a_{0}^{i}(t)}{2}+\sum_{n=1}^{\infty}\left[a_{n}^{i}(t) \cos n r s+b_{n}^{i}(t) \sin n r s\right]
$$

and

$$
\bar{B}^{j}(s)-\frac{s}{t} \bar{B}^{j}(t)=\frac{a_{0}^{j, b}(t)}{2}+\sum_{n=1}^{\infty}\left[a_{n}^{j, b}(t) \cos n r s+b_{n}^{j, b}(t) \sin n r s\right]
$$

where

$$
\begin{aligned}
& a_{n}^{i}(t)=\frac{2}{t} \int_{0}^{t}\left(\bar{W}^{i}(s)-\frac{s}{t} \bar{W}^{i}(t)\right) \cos n r s d s, \\
& b_{n}^{i}(t)=\frac{2}{t} \int_{0}^{t}\left(\bar{W}^{i}(s)-\frac{s}{t} \bar{W}^{i}(t)\right) \sin n r s d s
\end{aligned}
$$

and

$$
\begin{gathered}
a_{n}^{j, b}(t)=\frac{2}{t} \int_{0}^{t}\left(\bar{B}^{j}(s)-\frac{s}{t} \bar{B}^{j}(t)\right) \cos n r s d s, \\
b_{n}^{j, b}(t)=\frac{2}{t} \int_{0}^{t}\left(\bar{B}^{j}(s)-\frac{s}{t} \bar{B}^{j}(t)\right) \sin n r s d s
\end{gathered}
$$

for $n \geq 1$. The convergences in (4.4) and (4.5) are in $L^{2}(\Omega \times[0, t])$. It is easy to see that if $n \geq 1, a_{n}^{i}(t), b_{n}^{i}(t), a_{n}^{j, b}(t)$ and $b_{n}^{j, b}(t)$ are normally distributed with mean 0 and variance $t / 2 \pi^{2} n^{2}$ ([17], page 198). Furthermore, $\left\{a_{n}^{i}(t), b_{n}^{i}(t)\right\}$ and $\left\{a_{n}^{j, b}(t), b_{n}^{j, b}(t)\right\}$ are pairwise independent ([17], page 198). One can use wellknown random number generators to simulate these random coefficients (cf. [12], Section 3.1.2, [17], Section 1.3, and [18], Section 1.2).

LEMMA 4.1. Let $t_{0}, t \geq 0$. Then

$$
\begin{aligned}
& J_{i, j}\left(t_{0}, t_{0}+t ;-b\right) \\
& =\frac{1}{2}\left(\bar{W}^{i}(t) \bar{B}^{j}(t)\right)-\frac{1}{2}\left(\bar{W}^{i}(t) a_{0}^{j, b}\left(t_{0}\right)-\bar{B}^{j}(t) a_{0}^{i}\left(t_{0}\right)\right) \\
& \quad+\pi \sum_{n=1}^{\infty} n\left[a_{n}^{i}\left(t_{0}\right) b_{n}^{j, b}\left(t_{0}\right)-b_{n}^{i}\left(t_{0}\right) a_{n}^{j, b}\left(t_{0}\right)\right], \quad 1 \leq i, j \leq d,
\end{aligned}
$$

where the infinite series converges in $L^{2}(\Omega, \mathbf{R})$. 
ProOF. It suffices to show (4.8) for $t_{0}=0$. Fix $t>0$. For simplicity of notation, we write

$$
\begin{array}{rlrl}
a_{n}^{j} & :=a_{n}^{j}(0), & b_{n}^{j}:=b_{n}^{j}(0), \\
a_{n}^{j, b}:=a_{n}^{j, b}(0), & b_{n}^{j, b}:=b_{n}^{j, b}(0)
\end{array}
$$

and

$$
W_{N}^{i}(s):=\frac{s}{t} W^{i}(t)+\frac{a_{0}^{i}}{2}+\sum_{n=1}^{N}\left(a_{n}^{i} \cos n r s+b_{n}^{i} \sin n r s\right) .
$$

It is easy to check that

$$
\int_{b}^{t+b} \int_{0}^{s-b} \circ d W_{N}^{i}(v) \circ d W^{j}(s) \rightarrow \int_{b}^{t+b} \int_{0}^{s-b} \circ d W^{i}(v) \circ d W^{j}(s)
$$

in $L^{2}(\Omega)$ as $N \rightarrow \infty$. Then we may write

$$
\begin{aligned}
J_{i, j}(0, t ;-b)= & \int_{b}^{t+b} W^{i}(s-b) \circ d W^{j}(s) \\
= & \int_{b}^{t+b} \frac{(s-b)}{t} W^{i}(t) \circ d W^{j}(s)+\frac{a_{0}^{i}}{2} \bar{B}^{j}(t) \\
& +\sum_{n=1}^{\infty}\left[a_{n}^{i} \int_{b}^{t+b} \cos n r(s-b) d W^{j}(s)\right. \\
& \left.\quad+b_{n}^{i} \int_{b}^{t+b} \sin n r(s-b) d W^{j}(s)\right] .
\end{aligned}
$$

For any $n \geq 1$, we have

$$
\begin{aligned}
\int_{b}^{t+b} & \cos n r(s-b) d W^{j}(s) \\
= & \int_{0}^{t} \cos n r s d \bar{B}^{j}(s) \\
= & \int_{0}^{t} \cos n r s d\left(\bar{B}^{j}(s)-\frac{s}{t} \bar{B}^{j}(t)\right)+\int_{0}^{t} \cos n r s d\left(\frac{s}{t} \bar{B}^{j}(t)\right) \\
= & \left.\cos n r s\left(\bar{B}^{j}(s)-\frac{s}{t} \bar{B}^{j}(t)\right)\right|_{0} ^{t} \\
& +n r \int_{0}^{t}\left(\bar{B}^{j}(s)-\frac{s}{t} \bar{B}^{j}(t)\right) \sin n r s d s+\frac{\bar{B}^{j}(t)}{t} \int_{0}^{t} \cos n r s d s \\
= & \frac{t}{2} n r b_{n}^{j, b} .
\end{aligned}
$$


Similarly, we have

$$
\int_{b}^{t+b} \sin n r(s-b) d W^{j}(s)=-\frac{t}{2} n r a_{n}^{j, b} .
$$

So

$$
\begin{aligned}
J_{i, j}(0, t ;-b)= & \frac{W^{i}(t)}{t} \int_{0}^{t} s d \bar{B}^{j}(s) \\
& +\frac{a_{0}^{i}}{2} \bar{B}^{j}(t)+\pi \sum_{n=1}^{\infty} n\left(a_{n}^{i} b_{n}^{j, b}-b_{n}^{i} a_{n}^{j, b}\right) .
\end{aligned}
$$

Now,

$$
\begin{aligned}
\int_{0}^{t} s d \bar{B}^{j}(s) & =t \bar{B}^{j}(t)-\int_{0}^{t} \bar{B}^{j}(s) d s \\
& =\frac{t}{2} \bar{B}^{j}(t)-\int_{0}^{t}\left(\bar{B}^{j}(s)-\frac{s}{t} \bar{B}^{j}(t)\right) d s \\
& =\frac{t}{2}\left(\bar{B}^{j}(t)-a_{0}^{j, b}\right) .
\end{aligned}
$$

Therefore,

$$
\begin{gathered}
J_{i, j}(0, t ;-b)=\frac{1}{2} W^{i}(t) \bar{B}^{j}(t)-\frac{1}{2}\left(W^{i}(t) a_{0}^{j, b}-\bar{B}^{j}(t) a_{0}^{i}\right) \\
+\pi \sum_{n=1}^{\infty} n\left(a_{n}^{i} b_{n}^{j, b}-b_{n}^{i} a_{n}^{j, b}\right) .
\end{gathered}
$$

The expansion of $J_{i, j}(0, t ;-b)$ is a generalization of the expansion of

$$
\begin{aligned}
\int_{0}^{t} \int_{0}^{s} \circ d W^{i}(v) \circ d W^{j}(s) \\
=\frac{1}{2}\left(W^{i}(t) W^{j}(t)\right)-\frac{1}{2}\left[W^{i}(t) a_{0}^{j, b}-W^{j}(t) a_{0}^{i}\right] \\
\quad+\pi \sum_{n=1}^{\infty} n\left(a_{n}^{i} b_{n}^{j}-b_{n}^{i} a_{n}^{j}\right)
\end{aligned}
$$

(see $[11,17]$ and [18]). Set

$$
\begin{aligned}
J_{i, j}^{p}\left(t_{0}, t_{0}+t ;-b\right) & \\
:= & \frac{1}{2}\left(\bar{W}^{i}(t) \bar{B}^{j}(t)\right)-\frac{1}{2}\left[\bar{W}^{i}(t) a_{0}^{j, b}\left(t_{0}\right)-\bar{B}^{j}(t) a_{0}^{i}\left(t_{0}\right)\right] \\
& +\pi \sum_{n=1}^{p} n\left[a_{n}^{i}\left(t_{0}\right) b_{n}^{j, b}\left(t_{0}\right)-b_{n}^{i}\left(t_{0}\right) a_{n}^{j, b}\left(t_{0}\right)\right] .
\end{aligned}
$$

Then $J_{i, j}^{p}\left(t_{0}, t_{0}+t ;-b\right)$ can be used to approximate $J_{i, j}\left(t_{0}, t_{0}+t ;-b\right)$ in the mean 
square. The rate of convergence is given in Lemma 4.2.

LEMMA 4.2. For any integer $p \geq 1$ and $t>0$, we have

$$
E\left|J_{i, j}^{p}(0, t ;-b)-J_{i, j}(0, t ;-b)\right|^{2} \leq \frac{t^{2}}{2 \pi^{2} p} .
$$

ProOF. Let $p \geq 1$ be any integer. Then

$$
\sum_{n=p+1}^{\infty} \frac{1}{n^{2}} \leq \int_{p}^{\infty} \frac{1}{u^{2}} d u=\frac{1}{p}
$$

Since $a_{n}^{i}$ and $b_{n}^{i}$ are independent, $E\left(a_{n}^{i} b_{n}^{i}\right)=0$ and $E\left(a_{n}^{j, b} b_{n}^{j, b}\right)=0$, we have

$$
\begin{aligned}
E \mid J_{i, j}^{p} & (0, t ;-b)-\left.J_{i, j}(0, t ;-b)\right|^{2} \\
& =\pi^{2} \sum_{n=p+1}^{\infty} n^{2} E\left(a_{n}^{i} b_{n}^{j, b}-b_{n}^{i} a_{n}^{j, b}\right)^{2} \\
& =\pi^{2} \sum_{n=p+1}^{\infty} n^{2}\left[E\left(a_{n}^{i} b_{n}^{j, b}\right)^{2}+E\left(b_{n}^{i} a_{n}^{j, b}\right)^{2}\right] \\
& =\frac{t^{2}}{2 \pi^{2}} \sum_{n=p+1}^{\infty} \frac{1}{n^{2}} \\
& \leq \frac{t^{2}}{2 \pi^{2} p} .
\end{aligned}
$$

5. The strong Milstein scheme. In this section we construct a strong Milstein scheme of order 1 for the SDDE (1.6). Our construction relies heavily on the Itô formula for "tame" functions (Theorem 2.1).

Throughout this section, we assume that in (1.6) the coefficients $g \in C^{1,2}(T \times$ $\left.\mathbf{R}^{k_{1} m}, L\left(\mathbf{R}^{d}, \mathbf{R}^{m}\right)\right)$ and $h \in C^{1,2}\left(T \times \mathbf{R}^{k_{2} m}, \mathbf{R}^{m}\right)$. For convenience, set $W(s)=$ $W(0)=0$, for all $s \leq 0$. We also define

$$
\begin{aligned}
& u(t):= \begin{cases}g\left(t, \Pi_{1}\left(X_{t}\right)\right), & 0 \leq t \leq a, \\
0, & t<0,\end{cases} \\
& v(t):= \begin{cases}h\left(t, \Pi_{2}\left(X_{t}\right)\right), & 0 \leq t \leq a, \\
\eta(t), & t<0 .\end{cases}
\end{aligned}
$$

We first derive the Milstein scheme for the case $d=m=1$. 
5.1. Itô-Taylor expansion. Assume that $0<t_{0}<t$, and $\vec{x}=\left(x_{1}, \ldots, x_{k_{1}}\right) \in$ $\mathbf{R}^{k_{1}}$. Applying the Itô formula (2.10), we have

$$
\begin{aligned}
& g\left(t, \Pi_{1}\left(X_{t}\right)\right)-g\left(t_{0}, \Pi_{1}\left(X_{t_{0}}\right)\right) \\
& =\int_{t_{0}}^{t} \frac{\partial g}{\partial s}\left(s, \Pi_{1}\left(X_{s}\right)\right) d s \\
& \quad+\sum_{i=1}^{k_{1}} \int_{t_{0}+s_{1, i}}^{t+s_{1, i}} \frac{\partial g}{\partial x_{i}}\left(s-s_{1, i}, \Pi_{1}\left(X_{s-s_{1, i}}\right)\right) u(s) d W(s) \\
& +\sum_{i=1}^{k_{1}} \int_{t_{0}}^{t}\left[\frac{\partial g}{\partial x_{i}}\left(s, \Pi_{1}\left(X_{s}\right)\right) v\left(s+s_{1, i}\right)\right. \\
& \left.\quad+\frac{1}{2}\left\langle\frac{\partial^{2} g}{\partial x_{i}^{2}}\left(s, \Pi_{1}\left(X_{s}\right)\right) \nabla_{s_{1, i}}^{+} X(s), \nabla_{s_{1, i}}^{-} X(s)\right\rangle\right] d s,
\end{aligned}
$$

where $\nabla_{s_{1, i}}^{ \pm} X(s)$ are defined by (2.9). Applying the Itô formula (2.10) again and using similar notations for $h$, we obtain

$$
\begin{aligned}
& h\left(t, \Pi_{2}\left(X_{t}\right)\right)-h\left(t_{0}, \Pi_{2}\left(X_{t_{0}}\right)\right) \\
& =\int_{t_{0}}^{t} \frac{\partial h}{\partial s}\left(s, \Pi_{2}\left(X_{s}\right)\right) d s \\
& \quad+\sum_{i=1}^{k_{2}} \int_{t_{0}+s_{2, i}}^{t+s_{2, i}} \frac{\partial h}{\partial x_{i}}\left(s-s_{2, i}, \Pi_{2}\left(X_{s-s_{2, i}}\right)\right) u(s) d W(s) \\
& \quad+\frac{1}{2} \sum_{i=1}^{k_{2}} \int_{t_{0}}^{t}\left[\frac{\partial h}{\partial x_{i}}\left(s, \Pi_{2}\left(X_{s}\right)\right) v\left(s+s_{2, i}\right)\right. \\
& \left.\quad+\frac{1}{2}\left\langle\frac{\partial^{2} g}{\partial x_{i}^{2}}\left(s, \Pi_{2}\left(X_{s}\right)\right) \nabla_{s_{2, i}}^{+} X(s), \nabla_{s_{2, i}}^{-} X(s)\right\rangle\right] d s .
\end{aligned}
$$

Substituting (5.2) and (5.3) into (1.6), we get the following approximate (ItôTaylor) expansion of (1.6):

$$
\begin{aligned}
X(t)=X\left(t_{0}\right) & +g\left(t_{0}, \Pi_{1}\left(X_{t_{0}}\right)\right)\left[W(t)-W\left(t_{0}\right)\right]+h\left(t_{0}, \Pi_{2}\left(X_{t_{0}}\right)\right)\left(t-t_{0}\right) \\
+\sum_{i=1}^{k_{1}} & \frac{\partial g}{\partial x_{i}}\left(t_{0}, \Pi_{1}\left(X_{t_{0}}\right)\right) u\left(t_{0}+s_{1, i}\right) \\
& \times \int_{t_{0}}^{t} \int_{t_{0}+s_{1, i}}^{t_{1}+s_{1, i}} d W\left(t_{2}\right) d W\left(t_{1}\right)+R\left(t_{0}, t\right)
\end{aligned}
$$


where

$$
\begin{aligned}
R\left(t_{0}, t\right):= & \sum_{i=1}^{k_{1}}\left\{\int _ { t _ { 0 } } ^ { t } \int _ { t _ { 0 } + s _ { 1 , i } } ^ { t _ { 1 } + s _ { 1 , i } } \left[\frac{\partial g}{\partial x_{i}}\left(t_{2}-s_{1, i}, \Pi_{1}\left(X_{t_{2}-s_{1, i}}\right)\right) u\left(t_{2}\right)\right.\right. \\
& \left.\left.\quad-\frac{\partial g}{\partial x_{i}}\left(t_{0}, \Pi_{1}\left(X_{t_{0}}\right)\right) u\left(t_{0}+s_{1, i}\right)\right] d W\left(t_{2}\right) d W\left(t_{1}\right)\right\} \\
& +\int_{t_{0}}^{t} \int_{t_{0}}^{t_{1}} \sum_{i=1}^{k_{1}}\left[\frac{\partial g}{\partial x_{i}}\left(t_{2}, \Pi_{1}\left(X_{t_{2}}\right)\right) v\left(t_{2}+s_{1, i}\right)\right. \\
& +\sum_{i=1}^{k_{2}} \int_{t_{0}}^{t} \int_{t_{0}+s_{2, i}}^{t_{1}+s_{2, i}} \frac{\partial h}{\partial x_{i}}\left(t_{2}-s_{2, i}, \Pi_{2}\left(X_{t_{2}-s_{2, i}}\right)\right) u\left(t_{2}\right) d W\left(t_{2}\right) d t_{1} \\
& \left.+\int_{t_{0}}^{t} \int_{t_{0}}^{t_{1}} \sum_{i=1}^{k_{2}}\left[\frac{\partial h}{\partial x_{i}^{2}}\left(t_{2}, \Pi_{1}\left(X_{t_{2}}\right)\right) \nabla_{s_{1, i}}^{+} X_{t_{2}}, \nabla_{s_{1, i}}^{-} X_{t_{2}}\right)\right] d t_{2} d W\left(X_{1}\right) \\
& +\int_{t_{0}}^{t} \int_{t_{0}}^{t_{1}}\left[\frac{\partial g}{\partial t_{2}}\left(t_{2}, \Pi_{1}\left(x_{t_{2}}\right)\right)+s_{2, i}\right) \\
& \left.\left.\left.+\frac{1}{2}\left\langle\frac{\partial^{2} h}{\partial x_{i}^{2}}\left(t_{2}, \Pi_{2}\left(X_{t_{2}}\right)\right) \nabla_{s_{2, i}}^{+} X_{t_{2}}, \nabla_{s_{2, i}}^{-} X_{t_{2}}\right\rangle\right] d t_{2} d t_{1}, \Pi_{2}\left(X_{t_{2}}\right)\right)\right] d t_{2} d t_{1} .
\end{aligned}
$$

In the above expression, the stochastic integrals

$$
\int_{t_{0}+s_{1, i}}^{t_{1}+s_{1, i}} \frac{\partial g}{\partial x_{i}}\left(t_{2}-s_{1, i}, \Pi_{1}\left(X_{t_{2}-s_{1, i}}\right)\right) u\left(t_{2}\right) d W\left(t_{2}\right)
$$

and

$$
\int_{t_{0}+s_{2, i}}^{t_{1}+s_{2, i}} \frac{\partial h}{\partial x_{i}}\left(t_{2}-s_{2, i}, \Pi_{2}\left(X_{t_{2}-s_{2, i}}\right)\right) u\left(t_{2}\right) d W\left(t_{2}\right)
$$

are Skorohod integrals. Define

$$
I\left(t_{0}+s_{i, j}, t+s_{i, j} ; s_{i, j}\right):=\int_{t_{0}}^{t} \int_{t_{0}+s_{i, j}}^{t_{1}+s_{i, j}} d W\left(t_{2}\right) d W\left(t_{1}\right),
$$

for $i=1,2$ and $j=1, \ldots, k_{i}$. Recall the definition of $J\left(t_{0}+s_{i, j}, t+s_{i, j} ; s_{i, j}\right)$ in (4.1). Note that if $s_{i, j}<0$, then

$$
I\left(t_{0}+s_{i, j}, t+s_{i, j} ; s_{i, j}\right)=\int_{t_{0}}^{t} \int_{t_{0}+s_{i, j}}^{t_{1}+s_{i, j}} \circ d W\left(t_{2}\right) \circ d W\left(t_{1}\right)
$$


if $s_{i, j}=0$, then

$$
\begin{aligned}
I\left(t_{0}+s_{i, j}, t+s_{i, j} ; s_{i, j}\right) & =\int_{t_{0}}^{t}\left[W\left(t_{1}\right)-W\left(t_{0}\right)\right] d W\left(t_{1}\right) \\
& =\frac{\left(W(t)-W\left(t_{0}\right)\right)^{2}}{2}-\frac{t-t_{0}}{2} .
\end{aligned}
$$

5.2. The one-dimensional Milstein scheme $(d=m=1)$. Assume $d=m=1$. Let $\pi:-\tau=t_{-l}<\cdots<t_{0}=0<\cdots<t_{n}=a$ be a partition of $[-\tau, a]$. We introduce the Milstein scheme for the SDDE (1.6) as follows:

$$
\begin{aligned}
X^{\pi}(t)= & X^{\pi}\left(t_{k}\right)+h\left(t_{k}, \Pi_{2}\left(X_{t_{k}}^{\pi}\right)\right)\left(t-t_{k}\right)+g\left(t_{k}, \Pi_{1}\left(X_{t_{k}}^{\pi}\right)\right)\left(W(t)-W\left(t_{k}\right)\right) \\
& +\sum_{i=1}^{k_{1}} \frac{\partial g}{\partial x_{i}}\left(t_{k}, \Pi_{1}\left(X_{t_{k}}^{\pi}\right)\right) u^{\pi}\left(t_{k}+s_{1, i}\right) I\left(t_{k}+s_{1, i}, t+s_{1, i} ; s_{1, i}\right)
\end{aligned}
$$

for $t_{k}<t \leq t_{k+1}$, where

$$
u^{\pi}(t)= \begin{cases}g\left(t, \Pi_{1}\left(X_{t}^{\pi}\right)\right), & t \geq 0, \\ 0, & -\tau \leq t<0,\end{cases}
$$

and

$$
I\left(t_{k}+s_{1, i}, t+s_{1, i} ; s_{1, i}\right)=\int_{t_{k}}^{t} \int_{t_{k}+s_{1, i}}^{t_{1}+s_{1, i}} \circ d W\left(t_{2}\right) \circ d W\left(t_{1}\right) .
$$

Recall the notation

$$
\lfloor s\rfloor:= \begin{cases}t_{k}, & t_{k} \leq s<t_{k+1}, \\ t_{n_{t}}, & t_{n_{t}} \leq s \leq t,\end{cases}
$$

and introduce the following notation:

$$
\lceil s\rceil= \begin{cases}t_{k+1}, & t_{k}<s \leq t_{k+1}, \\ t, & t_{n_{t}}<s \leq t .\end{cases}
$$

In view of (5.7) and Lemma 4.2, we will use $J^{p}\left(t_{i}, t ; s_{1, i}\right)$ to approximate $I\left(t_{i}, t ; s_{1, i}\right)$.

Denote by

$$
Z^{\pi}(t):=X^{\pi}(t)-X(t), \quad t \in[-\tau, a]
$$

the global truncation error for the Milstein scheme, with $X$ the unique solution of the SDDE (1.6).

LEMMA 5.1. In the SDDE (1.6) (with $d=m=1$ ), suppose that $g \in$ $C_{b}^{2}\left(\mathbf{R}^{k_{1}}, \mathbf{R}\right), h \in C_{b}^{2}\left(\mathbf{R}^{k_{2}}, \mathbf{R}\right)$, have bounded first and second derivatives. Then for 
each integer $p \geq 1$, there exists a constant $K(p)>0$ such that

$$
\begin{aligned}
& E \mid\left\langle\frac { \partial ^ { 2 } g } { \partial \vec { x } ^ { 2 } } \left( s,\left.\Pi_{1}\left(X_{s}\right)\left|\nabla_{s_{1, i}}^{+} X_{s}, \nabla_{s_{1, i}}^{-} X_{s}\right\rangle\right|^{p} \leq K(p),\right.\right. \\
& E\left|\left\langle\frac{\partial^{2} h}{\partial \vec{x}^{2}}\left(s, \Pi_{2}\left(X_{s}\right)\right) \nabla_{s_{2, i}}^{+} X_{s}, \nabla_{s_{2, i}}^{-} X_{s}\right\rangle\right|^{p} \leq K(p),
\end{aligned}
$$

for all $t \in[0, a]$.

ProOF. By the definition of $\nabla_{s_{2, i}}^{ \pm} X(s)$ [see (2.9)], we have

$$
\begin{aligned}
\nabla_{s_{1, i}, s_{1, j}}^{+} X(s)= & 2 u\left(s+s_{1, i}\right) I_{\left\{s_{1, i}<s_{1, j}\right\}}+u\left(s+s_{1, i}\right) \delta_{i j} \\
& +2 \int_{0}^{s+s_{1, j}} D_{s+s_{1, i}} u(r) d W(r)+2 \int_{0}^{s+s_{1, j}} D_{s+s_{1, i}} v(r) d r
\end{aligned}
$$

and

$$
\nabla_{s_{1, i}, s_{1, j}}^{-} X(s)=u\left(s+s_{1, i}\right) \delta_{i j}
$$

Therefore,

$$
\begin{aligned}
&\left\langle\frac{\partial^{2} g}{\partial \vec{x}^{2}}\left(s, \Pi_{1}\left(X_{s}\right)\right) \nabla_{s_{1, i}}^{+} X(s), \nabla_{s_{1, i}}^{-} X(s)\right\rangle \\
&=2 \sum_{i=1}^{k_{1}}\left\{\frac{\partial^{2} g}{\partial x_{i} \partial x_{j}}\left(s, \Pi_{1}\left(X_{s}\right)\right) u\left(s+s_{1, i}\right)\right. \\
& \times\left[u\left(s+s_{1, i}\right) I_{\left\{s_{1, i}<s_{1, j}\right\}}+\frac{1}{2} u\left(s+s_{1, i}\right) \delta_{i j}\right. \\
&+\int_{0}^{s+s_{1, j}} D_{s+s_{1, i}} u(r) d W(r) \\
&\left.\left.+\int_{0}^{s+s_{1, j}} D_{s+s_{1, i}} v(r) d r\right]\right\} .
\end{aligned}
$$

If $r>0$, then

$$
\begin{aligned}
D_{s} u(r) & =D_{s} g\left(\Pi_{1}\left(X_{r}\right)\right) \\
& =\sum_{i=1}^{k_{1}} \frac{\partial g}{\partial x_{i}}\left(r, \Pi_{1}\left(X_{r}\right)\right) D_{s} X\left(r+s_{1, i}\right)
\end{aligned}
$$

and

$$
\begin{aligned}
D_{t} D_{s} u(r)= & \sum_{i, j=1}^{k_{1}} \frac{\partial^{2} g}{\partial x_{i} \partial x_{j}}\left(r, \Pi_{1}\left(X_{r}\right)\right) D_{s} X\left(r+s_{1, i}\right) D_{t} X\left(r+s_{1, j}\right) \\
& +\sum_{i=1}^{k_{1}} \frac{\partial g}{\partial x_{i}}\left(r, \Pi_{1}\left(X_{r}\right)\right) D_{t} D_{s} X\left(r+s_{1, i}\right)
\end{aligned}
$$


By Proposition 3.1 and Proposition 3.3, there exists a constant $C_{1}>0$ such that

$$
\begin{array}{r}
\sup _{0 \leq s \leq a} E\left(\sup _{s \leq r \leq a}\left|D_{s} X(r)\right|^{2}\right) \leq C_{1}, \\
\sup _{0 \leq s, t \leq a} E\left(\sup _{s \vee t \leq r \leq a}\left|D_{t} D_{s} X(r)\right|^{2}\right) \leq C_{1} .
\end{array}
$$

Since $g$ has bounded first and second derivatives, then there is a positive constant $C_{2}$ such that

$$
\begin{array}{rl}
\sup _{0 \leq s \leq a} & E\left(\sup _{s \leq r \leq a}\left|D_{s} u(r)\right|^{2}\right) \\
\quad \leq C_{2} k_{1} \sup _{0 \leq s \leq a} E\left(\sup _{s \leq r \leq a}\left|D_{s} X(r)\right|^{2}\right) \leq C_{1} C_{2} k_{1}
\end{array}
$$

and

$$
\sup _{0 \leq s, t \leq a} E\left(\sup _{s \vee t \leq r \leq a}\left|D_{t} D_{s} u(r)\right|^{2}\right) \leq C_{1}^{2} C_{2}^{2} k_{1}+C_{1} C_{2} k_{1} .
$$

If $r<s+s_{1, i}$, then

$$
\begin{aligned}
& D_{s+s_{1, i}} u(r)=0, \\
& D_{s+s_{1, i}} v(r)=0 .
\end{aligned}
$$

Therefore,

$$
\begin{aligned}
& E\left(\int_{t+s_{1, i}}^{t+s_{1, j}} D_{t+s_{1, i}} u(r) d W(r)\right)^{2} \\
& \quad \leq \int_{t+s_{1, i}}^{t+s_{1, j}} \int_{t+s_{1, i}}^{t+s_{1, j}} E\left(D_{s} D_{t+s_{1, i}} u(r)\right)^{2} d r d s+\int_{t+s_{1, i}}^{t+s_{1, j}} E\left(D_{t+s_{1, i}} u(r)\right)^{2} d r \\
& \quad \leq C_{2} k_{1}^{2} C_{1}^{2}+2 C_{2} k_{1} C_{1} \\
& \quad=: K_{1} .
\end{aligned}
$$

Similarly, there exists a constant $K_{2}>0$ such that

$$
E\left(\int_{t+s_{1, i}}^{t+s_{1, j}} D_{t+s_{1, i}} v(r) d r\right)^{2} \leq K_{2}
$$

So the first inequality of (5.10) follows from the above two inequalities and the Lipschitz and bounded conditions on $h, g$ [(1.4) and (1.5)]. The second estimate of (5.10) is proved by a similar argument.

THEOREM 5.2. Consider the Milstein scheme (5.9) for the SDDE (1.6). Recall that $Z^{\pi}:=X^{\pi}-X$ is the global truncation error for any partition $\pi$ of $[-\tau, a]$. 
Let $0<\gamma \leq 1$. Suppose that $\eta:[-\tau, 0] \rightarrow \mathbf{R}^{m}$ is of bounded variation and is $\left(\frac{\gamma}{2}\right)$-Hölder continuous. Let $g \in C^{1,2}\left(T \times \mathbf{R}^{k_{1}}, \mathbf{R}\right), h \in C^{1,2}\left(T \times \mathbf{R}^{k_{2}}, \mathbf{R}\right)$ have bounded first and second spatial derivatives. Assume that

$$
\sup _{-\tau \leq s \leq 0}\left|Z^{\pi}(s)\right| \leq C^{\prime}|\pi|^{\gamma}
$$

for some positive constant $C^{\prime}$. Then there exists a constant $C>0$ (depending on a and independent of $\pi$ ) such that

$$
\sup _{-\tau \leq s \leq a} E\left|Z^{\pi}(s)\right|^{2} \leq C|\pi|^{2 \gamma} .
$$

PROOF. We express the global error in the form

$$
Z^{\pi}(t)=Z^{\pi}(0)+I^{\pi}(t)-R^{\pi}(t),
$$

where

$$
\begin{aligned}
I^{\pi}(t)= & \sum_{i=1}^{n_{t}}\left[h\left(t_{i-1}, \Pi_{2}\left(X_{t_{i-1}}^{\pi}\right)\right)-h\left(t_{i-1}, \Pi_{2}\left(X_{t_{i-1}}\right)\right)\right]\left(t_{i}-t_{i-1}\right) \\
& +\sum_{i=1}^{n_{t}}\left[g\left(t_{i-1}, \Pi_{1}\left(X_{t_{i-1}}^{\pi}\right)\right)-g\left(t_{i-1}, \Pi_{1}\left(X_{t_{i-1}}\right)\right)\right]\left(W_{t_{i}}-W_{t_{i-1}}\right) \\
& +\left[h\left(t_{n_{t}}, \Pi_{2}\left(X_{t_{n_{t}}}^{\pi}\right)\right)-h\left(t_{n_{t}}, \Pi_{2}\left(X_{t_{n_{t}}}\right)\right)\right]\left(t-t_{n_{t}}\right) \\
& +\left[g\left(t_{n_{t}}, \Pi_{1}\left(X_{t_{n_{t}}}^{\pi}\right)\right)-g\left(t_{n_{t}}, \Pi_{1}\left(X_{t_{n_{t}}}\right)\right)\right]\left(W(t)-W\left(t_{n_{t}}\right)\right) \\
& +\sum_{i=1}^{n_{t}} \sum_{j=1}^{k_{1}}\left\{I ( t _ { i - 1 } , t _ { i } ; s _ { 1 , j } ) \left[\frac{\partial g}{\partial x_{j}}\left(t_{i-1}, \Pi_{1}\left(X_{t_{i-1}}^{\pi}\right)\right) u^{\pi}\left(t_{i-1}+s_{1, j}\right)\right.\right. \\
& +\sum_{j=1}^{k_{1}}\left\{I ( t _ { n _ { t } } , t ; s _ { 1 , j } ) \left[\frac{\partial g}{\partial x_{j}}\left(t_{n_{t}}, \Pi_{1}\left(X_{t_{n_{t}}}^{\pi}\right)\right) u^{\pi}\left(t_{n_{t}}+s_{1, j}\right)\right.\right. \\
& \left.\left.\left.-\frac{\partial g}{\partial x_{j}}\left(t_{n_{t}}, \Pi_{1-1}, \Pi_{1}\left(X_{t_{n_{t}}}\right)\right) u\left(X_{t_{i-1}}\right)\right) u\left(t_{i-1}+s_{1, j}\right)\right]\right\}
\end{aligned}
$$

and

$$
R^{\pi}(t)=\sum_{i=1}^{n_{t}} R\left(t_{i-1}, t_{i}\right)+R\left(t_{n_{t}}, t\right) .
$$

We shall decompose $R^{\pi}(t)$ into five parts:

$$
R^{\pi}(t)=R_{1}^{\pi}(t)+R_{2}^{\pi}(t)+R_{3}^{\pi}(t)+R_{4}^{\pi}(t)+R_{5}^{\pi}(t),
$$


where

$$
\begin{aligned}
& R_{1}^{\pi}(t):=\sum_{i=1}^{n_{t}} \sum_{j=1}^{k_{1}}\left\{\int _ { t _ { i - 1 } } ^ { t _ { i } } \int _ { t _ { i - 1 } + s _ { 1 , j } } ^ { s + s _ { 1 , j } } \left[\frac{\partial g}{\partial x_{j}}\left(r-s_{1, j}, \Pi_{1}\left(X_{r-s_{1, j}}\right)\right) u(r)\right.\right. \\
& -\frac{\partial g}{\partial x_{j}}\left(t_{i-1}, \Pi_{1}\left(X_{t_{i-1}}\right)\right) \\
& \left.\left.\times u\left(t_{i-1}+s_{1, j}\right)\right] d W(r) d W(s)\right\} \\
& +\sum_{j=1}^{k_{1}}\left\{\int _ { t _ { n } } ^ { t } \int _ { t _ { n _ { t } } + s _ { 1 , j } } ^ { s + s _ { 1 , j } } \left[\frac{\partial g}{\partial x_{j}}\left(r-s_{1, j}, \Pi_{1}\left(X_{r-s_{1, j}}\right)\right) u(r)\right.\right. \\
& \left.\left.-\frac{\partial g}{\partial x_{j}}\left(t_{n_{t}}, \Pi_{1}\left(X_{t_{n_{t}}}\right)\right) u\left(t_{n_{t}}+s_{1, j}\right)\right] d W(r) d W(s)\right\} \\
& =\sum_{j=1}^{k_{1}}\left\{\int _ { 0 } ^ { t } \int _ { \lfloor s \rfloor + s _ { 1 , j } } ^ { s + s _ { 1 , j } } \left[\frac{\partial g}{\partial x_{j}}\left(r-s_{1, j}, \Pi_{1}\left(X_{r-s_{1, j}}\right)\right) u(r)\right.\right. \\
& \left.\left.-\frac{\partial g}{\partial x_{j}}\left(\lfloor s\rfloor, \Pi_{1}\left(X_{\lfloor s\rfloor}\right)\right) u\left(\lfloor s\rfloor+s_{1, j}\right)\right] d W(r) d W(s)\right\}, \\
& R_{2}^{\pi}(t):=\sum_{j=1}^{k_{1}} \int_{0}^{t} \int_{\lfloor s\rfloor}^{s}\left[\frac{\partial g}{\partial x_{j}}\left(r, \Pi_{1}\left(X_{r}\right)\right) v\left(r+s_{1, j}\right)\right. \\
& \left.+\frac{1}{2}\left\langle\frac{\partial^{2} g}{\partial \vec{x}^{2}}\left(r, \Pi_{1}\left(X_{r}\right)\right) \nabla_{s_{1, j}}^{+} X_{r}, \nabla_{s_{1, j}}^{-} X_{r}\right\rangle\right] d r d W(s), \\
& R_{3}^{\pi}(t):=\sum_{j=1}^{k_{2}} \int_{0}^{t} \int_{\lfloor s\rfloor+s_{2, j}}^{s+s_{2, j}} \frac{\partial h}{\partial x_{j}}\left(r-s_{2, j}, \Pi_{2}\left(X_{r-s_{2, j}}\right)\right) u(r) d W(r) d s, \\
& R_{4}^{\pi}(t):=\sum_{j=1}^{k_{2}} \int_{0}^{t} \int_{\lfloor s\rfloor}^{s}\left[\frac{\partial h}{\partial x_{j}}\left(r, \Pi_{2}\left(X_{r}\right)\right) v\left(r+s_{2, j}\right)\right. \\
& \left.+\frac{1}{2}\left\langle\frac{\partial^{2} h}{\partial \vec{x}^{2}}\left(r, \Pi_{2}\left(X_{r}\right)\right) \nabla_{s_{2, j}}^{+} X_{r}, \nabla_{s_{2, j}}^{-} X_{r}\right\rangle\right] d r d s
\end{aligned}
$$

and

$$
R_{5}^{\pi}(t):=\int_{0}^{t} \int_{\lfloor s\rfloor}^{s}\left\{\frac{\partial h}{\partial r}\left(r, \Pi_{2}\left(X_{r}\right)\right)+\frac{\partial g}{\partial r}\left(r, \Pi_{1}\left(X_{r}\right)\right)\right\} d r d s
$$


By the Itô isometry and the formula for the covariance between two Skorohod integrals ([22], Section 1.3.1), we have

$$
\begin{aligned}
& \sup E\left|R_{1}^{\pi}(s)\right|^{2} \\
& 0 \leq s \leq t \\
& \begin{aligned}
\leq k_{1} \sum_{j=1}^{k_{1}} E \int_{0}^{t}\left\{\int_{\lfloor s\rfloor+s_{1, j}}^{s+s_{1, j}}\right. & \frac{\partial g}{\partial x_{j}}\left(r-s_{1, j}, \Pi_{1}\left(X_{r-s_{1, j}}\right)\right) u(r) \\
& \left.\left.-\frac{\partial g}{\partial x_{j}}\left(\lfloor s\rfloor, \Pi_{1}\left(X_{\lfloor s\rfloor}\right)\right) u\left(\lfloor s\rfloor+s_{1, j}\right)\right] d W(r)\right\}^{2} d s
\end{aligned} \\
& \leq k_{1} \sum_{j=1}^{k_{1}} \int_{0}^{t} \int_{\lfloor s\rfloor+s_{1, j}}^{s+s_{1, j}} E\left[\frac{\partial g}{\partial x_{j}}\left(r-s_{1, j}, \Pi_{1}\left(X_{r-s_{1, j}}\right)\right) u(r)\right. \\
& \left.-\frac{\partial g}{\partial x_{j}}\left(\lfloor s\rfloor, \Pi_{1}\left(X_{\lfloor s\rfloor}\right)\right) u\left(\lfloor s\rfloor+s_{1, j}\right)\right]^{2} d r d s \\
& +k_{1} \sum_{j=1}^{k_{1}} \int_{0}^{t} \int_{\lfloor s\rfloor+s_{1, j}}^{s+s_{1, j}} \int_{\lfloor s\rfloor+s_{1, j}}^{s+s_{1, j}} E\left\{D _ { r _ { 1 } } \left[\frac { \partial g } { \partial x _ { j } } \left(r-s_{1, j},\right.\right.\right. \\
& \left.\Pi_{1}\left(X_{r-s_{1, j}}\right)\right) u(r) \\
& -\frac{\partial g}{\partial x_{j}}\left(\lfloor s\rfloor, \Pi_{1}\left(X_{\lfloor s\rfloor}\right)\right) \\
& \left.\left.\times u\left(\lfloor s\rfloor+s_{1, j}\right)\right]\right\}^{2} d r_{1} d r_{2} d s \\
& =k_{1} R_{11}^{\pi}(t)+k_{1} R_{12}^{\pi}(t) .
\end{aligned}
$$

By assumption, the function

$$
G_{j}(s, x, z)=\frac{\partial g}{\partial x_{j}}(s, x) g\left(s+s_{1, j}, z\right), \quad x \in \mathbf{R}^{k_{1}} \quad \text { and } \quad z \in \mathbf{R}^{k_{1}}
$$

is Lipschitz; that is, there exists a constant $L_{1}>0$ such that

$$
\left|G_{j}(s, z)-G_{j}(s, w)\right| \leq L_{1}|z-w| \quad \forall(z, w) \in \mathbf{R}^{2 k_{1}} \quad \text { and } \quad 1 \leq j \leq k_{1} .
$$

Using

$$
u(r)= \begin{cases}g\left(r, \Pi_{1}\left(X_{r}\right)\right), & r \geq 0 \\ 0, & r<0\end{cases}
$$

and

$$
\sup _{-\tau \leq r_{1} \leq \alpha<\beta \leq r_{2} \leq a} E|X(\beta)-X(\alpha)|^{2} \leq C_{2}\left|r_{2}-r_{1}\right|^{\gamma},
$$


it follows that

$$
\begin{aligned}
& R_{11}^{\pi}(t) \leq \sum_{j=1}^{k_{1}} \int_{0}^{t} \int_{\lfloor s\rfloor+s_{1, j}}^{s+s_{1, j}} E\left[G_{j}\left(r-s_{1, j}, \Pi_{1}\left(X_{r-s_{1, j}}\right), \Pi_{1}\left(X_{r}\right)\right)\right. \\
&\left.-G_{j}\left(\lfloor s\rfloor, \Pi_{1}\left(X_{\lfloor s\rfloor}\right), \Pi_{1}\left(X_{\lfloor s\rfloor+s_{1, j}}\right)\right)\right]^{2} \\
& \times I_{\left\{\lfloor s\rfloor+s_{1, j} \geq 0\right\}} d r d s \\
& \leq 2 k_{1} L_{1}^{2} \sum_{j=1}^{k_{1}} \int_{0}^{t} \int_{\lfloor s\rfloor+s_{1, j}-1 \leq r_{1}<r_{2} \leq a,\left|r_{2}-r_{1}\right| \leq|\pi|}^{s+s_{1, j}} E\left|X\left(r_{2}\right)-X\left(r_{1}\right)\right|^{2} d r d s \\
& \leq 2(a+1) k_{1}^{2} L_{1}^{2} C_{2}|\pi|^{2 \gamma} .
\end{aligned}
$$

Now for all $r \geq 0$ and $1 \leq j \leq k_{1}$,

$$
\begin{aligned}
& D_{s}\left(\frac{\partial g}{\partial x_{j}}\left(r-s_{1, j}, \Pi_{1}\left(X_{r-s_{1, j}}\right)\right) u(r)\right) \\
& \quad=g\left(r, \Pi_{1}\left(X_{r}\right)\right) \sum_{i=1}^{k_{1}} \frac{\partial^{2} g}{\partial x_{j} \partial x_{i}}\left(r-s_{1, j}, \Pi_{1}\left(X_{r-s_{1, j}}\right)\right) D_{s} X\left(r-s_{1, j}+s_{1, i}\right) \\
& \quad+\frac{\partial g}{\partial x_{j}}\left(r-s_{1, j}, \Pi_{1}\left(X_{r-s_{1, j}}\right)\right) \sum_{i=1}^{k_{1}} \frac{\partial g}{\partial x_{i}}\left(r, \Pi_{1}\left(X_{r}\right)\right) D_{s} X\left(r+s_{1, i}\right) .
\end{aligned}
$$

By Proposition 3.1, there exists a constant $C_{3}>0$ such that

$$
\sup _{0 \leq r \leq a} E\left(\sup _{0 \leq s \leq a}\left|D_{r} X(s)\right|^{2}\right) \leq C_{3} .
$$

By (1.8), (1.10) and boundedness of the spatial derivatives of $g$, there exists a constant $C_{4}>0$ such that

$$
\sup _{0 \leq r \leq a} \sup _{0 \leq s \leq a} E\left(\left|D_{s}\left(\frac{\partial g}{\partial x_{j}}\left(r-s_{1, j}, \Pi_{1}\left(X_{r-s_{1, j}}\right)\right) u(r)\right)\right|^{2}\right) \leq 2 C_{4} k_{1}^{2} .
$$

Therefore

$$
\begin{aligned}
R_{12}^{\pi}(t) & \leq k_{1} \sum_{j=1}^{k_{1}} \int_{0}^{t} \int_{\lfloor s\rfloor+s_{1, j}}^{s+s_{1, j}} \int_{\lfloor s\rfloor+s_{1, j}}^{s+s_{1, j}} 4 C_{4} k_{1}^{2} d r_{1} d r_{2} d s \\
& \leq 4(a+1) C_{4} k_{1}^{4}|\pi|^{2}
\end{aligned}
$$

Hence there is a constant $C_{5}>0$ such that

$$
\sup _{0 \leq s \leq t} E\left|R_{1}^{\pi}(s)\right|^{2} \leq C_{5}|\pi|^{2 \gamma}
$$


Applying Fubini's theorem, we can rewrite $R_{3}^{\pi}(t)$ as

$$
\begin{aligned}
R_{3}^{\pi}(t)= & \sum_{i=1}^{n_{t}} \sum_{j=1}^{k_{2}} \int_{t_{i-1}}^{t_{i}} \int_{t_{i-1}+s_{2, j}}^{s+s_{2, j}} \frac{\partial h}{\partial x_{j}}\left(r-s_{2, j}, \Pi_{2}\left(X_{r-s_{2, j}}\right)\right) u(r) d W(r) d s \\
& +\sum_{j=1}^{k_{2}} \int_{t_{n_{t}}}^{t} \int_{t_{n_{t}}+s_{2,1}}^{s+s_{2, j}} \frac{\partial h}{\partial x_{j}}\left(r-s_{2, j}, \Pi_{2}\left(X_{r-s_{2, j}}\right)\right) u(r) d W(r) d s .
\end{aligned}
$$

So we have

$$
\begin{aligned}
R_{3}^{\pi}(t)= & \sum_{i=1}^{n_{t}} \sum_{j=1}^{k_{2}} \int_{t_{i-1}+s_{2, j}}^{t_{i}+s_{2, j}} \int_{r-s_{2, j}}^{t_{i}} \frac{\partial h}{\partial x_{j}}\left(r-s_{2, j}, \Pi_{2}\left(X_{r-s_{2, j}}\right)\right) u(r) d s d W(r) \\
& +\sum_{j=1}^{k_{2}} \int_{t_{n_{t}}+s_{2, j}}^{t+s_{2, j}} \int_{r-s_{2, j}}^{t} \frac{\partial h}{\partial x_{j}}\left(r-s_{2, j}, \Pi_{2}\left(X_{r-s_{2, j}}\right)\right) u(r) d s d W(r) \\
= & \sum_{i=1}^{n_{t}} \sum_{j=1}^{k_{2}} \int_{t_{i-1}+s_{2, j}}^{t_{i}+s_{2, j}}\left(t_{i}+s_{2, j}-r\right) \frac{\partial h}{\partial x_{j}}\left(r-s_{2, j}, \Pi_{2}\left(X_{r-s_{2, j}}\right)\right) u(r) d W(r) \\
& +\sum_{j=1}^{k_{2}} \int_{t_{n_{t}}+s_{2, j}}^{t+s_{2, j}}\left(t+s_{2, j}-r\right) \frac{\partial h}{\partial x_{j}}\left(r-s_{2, j}, \Pi_{2}\left(X_{r-s_{2, j}}\right)\right) u(r) d W(r) \\
= & \sum_{j=1}^{k_{2}} \int_{s_{2, j}}^{t+s_{2, j}}\left(\left\lceil r-s_{2, j}\right\rceil+s_{2, j}-r\right) \frac{\partial h}{\partial x_{j}}\left(r-s_{2, j}, \Pi_{2}\left(X_{r-s_{2, j}}\right)\right) u(r) d W(r) .
\end{aligned}
$$

Applying the formula for covariance between two Skorohod integrals ([22], Section 1.3.1) and Proposition 3.1, we can show that there exists a constant $C_{6}>0$ such that

$$
\sup _{0 \leq s \leq t} E\left|R_{3}^{\pi}(s)\right|^{2} \leq C_{6}|\pi|^{2} .
$$

Similarly, by Lemma 5.1, we can easily show that there exist $C_{7}>0$ such that

$$
\begin{aligned}
& \sup _{0 \leq s \leq t} E\left|R_{2}^{\pi}(s)\right|^{2} \leq C_{7}|\pi|^{2}, \\
& \sup _{0 \leq s \leq t} E\left|R_{4}^{\pi}(s)\right|^{2} \leq C_{7}|\pi|^{2}, \\
& \sup _{0 \leq s \leq t} E\left|R_{5}^{\pi}(s)\right|^{2} \leq C_{7}|\pi|^{2} .
\end{aligned}
$$

By arguments similar to the ones used in the proof of Theorem 3.4 in [15], we obtain the following inequality:

$$
\sup _{0 \leq u \leq t} E\left|I^{\pi}(u)\right|^{2} \leq C_{1} \int_{0}^{t} \sup _{-\tau \leq u \leq s} E\left(\left|Z^{\pi}(u)\right|^{2}\right) d s
$$


for some constant $C_{1}>0$. From (5.16)-(5.19), there exist $C_{8}>0$ and $C_{9}>0$ such that

$$
\sup _{0 \leq u \leq t} E\left|Z^{\pi}(u)\right|^{2} \leq E\left|Z^{\pi}(0)\right|^{2}+C_{8}|\pi|^{2 \gamma}+C_{9} \int_{0}^{t} \sup _{-\tau \leq u \leq s} E\left|Z^{\pi}(u)\right|^{2} d s .
$$

So

$$
\sup _{-\tau \leq u \leq t} E\left|Z^{\pi}(u)\right|^{2} \leq\left(2 C^{\prime}+C_{8}\right)|\pi|^{2 \gamma}+C_{9} \int_{0}^{t} \sup _{-\tau \leq u \leq s} E\left|Z^{\pi}(u)\right|^{2} d s .
$$

By Gronwall's lemma, there exists a constant $C>0$ such that

$$
E \sup _{-\tau \leq s \leq t}\left|Z^{\pi}(s)\right|^{2} \leq C|\pi|^{2 \gamma}
$$

REMARKS.

1. Let us consider a particular case when $g$ and $h$ are of the form

$$
\begin{aligned}
& g\left(s, \Pi_{1}\left(X_{s}\right)\right)=\sum_{i=1}^{k_{1}} a_{i}\left(s, X_{s}\left(s_{1, i}\right)\right), \\
& h\left(s, \Pi_{2}\left(X_{s}\right)\right)=\sum_{j=1}^{k_{2}} b_{j}\left(s, X_{s}\left(s_{2, j}\right)\right),
\end{aligned}
$$

where $a_{i}, b_{j} \in C_{b}^{1,2}(T \times R)$ for $1 \leq i \leq k_{1}$ and $1 \leq j \leq k_{2}$. In this case, one may also apply the nonanticipating Itô formula to

$$
\begin{aligned}
& a_{i}\left(t+s_{1,1}, X\left(t+s_{1,1}\right)\right)-a_{i}\left(t_{0}+s_{1,1}, X\left(t_{0}+s_{1,1}\right)\right), \\
& a_{i}\left(t+s_{1,1}, X\left(t+s_{1,1}\right)\right)-a_{i}\left(t_{0}+s_{1,1}, X\left(t_{0}+s_{1,1}\right)\right)
\end{aligned}
$$

to prove Theorem 5.2 (cf. [28]).

2. One can allow the initial process $\eta$ to be a sample continuous semimartingale in the following way. Replace $W$ by an extended Brownian motion $W(t)$, $t \geq-\tau$, with the associated Brownian filtration $\left(\mathcal{F}_{t}\right)_{-\tau \leq t \leq a}$. Assume that $\eta(t) \in \mathbb{D}_{m}^{1, \infty}$ for all $t \in[-\tau, 0]$, and $\eta$ is an $\left(\mathcal{F}_{t}\right)_{-\tau \leq t \leq 0}$-continuous semimartingale satisfying

$$
\begin{gathered}
\sup _{-\tau \leq \alpha<\beta \leq 0} E|\eta(\beta)-\eta(\alpha)|^{2} \leq C_{2}|\beta-\alpha|^{\gamma}, \\
\sup _{-\tau \leq s \leq 0} E\left(\left|Z^{\pi}(s)\right|^{2}\right) \leq C^{\prime}|\pi|^{2 \gamma}
\end{gathered}
$$

for some positive constants $C_{2}$ and $C^{\prime}$. The arguments in Section 2 and the proof of Theorem 5.2 may be adapted to include this generalization. 
We can rewrite the SDDE (1.6) in Stratonovich form, namely, if $t \geq 0$,

$$
\begin{aligned}
X(t)= & \eta(0)+\int_{0}^{t} g\left(s, \Pi_{1}\left(X_{s}\right)\right) \circ d W(s) \\
& +\int_{0}^{t}\left[h\left(s, \Pi_{2}\left(X_{s}\right)\right)-\frac{1}{2} \frac{\partial g}{\partial x_{k_{1}}}\left(s, \Pi_{1}\left(X_{s}\right)\right) g\left(s, \Pi_{1}\left(X_{s}\right)\right)\right] d s,
\end{aligned}
$$

if $s_{k_{1}}=0$. If $s_{k_{1}}<0$, then the SDDE is of the same form as (1.6) except the Itô integral is replaced by a Stratonovich integral, that is,

$$
X(t)=\eta(0)+\int_{0}^{t} g\left(s, \Pi_{1}\left(X_{s}\right)\right) \circ d W(s)+\int_{0}^{t} h\left(s, \Pi_{2}\left(X_{s}\right)\right) d s .
$$

Bell and Mohammed ([5, 6]) derived a similar result in the case of a single delay. From Corollary 2.5, we can obtain the following Stratonovich-Taylor expansion of $X(t)$ (cf. [28]):

$$
\begin{aligned}
X(t)=X\left(t_{0}\right) & +g\left(t_{0}, \Pi_{1}\left(X_{t_{0}}\right)\right)\left[W(t)-W\left(t_{0}\right)\right] \\
& +\bar{h}\left(t_{0}, \Pi_{2}\left(X_{t_{0}}\right)\right)\left(t-t_{0}\right) \\
& +\sum_{i=1}^{k_{1}} \frac{\partial g}{\partial x_{i}}\left(t_{0}, \Pi_{1}\left(X_{t_{0}}\right)\right) u\left(t_{0}+s_{1, i}\right) \\
& \times \int_{t_{0}}^{t} \int_{t_{0}+s_{1, i}}^{t_{1}+s_{1, i}} \circ d W\left(t_{2}\right) \circ d W\left(t_{1}\right)+\bar{R}\left(t_{0}, t\right),
\end{aligned}
$$

where

$\bar{R}\left(t_{0}, t\right)$

$$
\begin{aligned}
=\sum_{i=1}^{k_{1}}\left\{\int _ { t _ { 0 } } ^ { t } \int _ { t _ { 0 } + s _ { 1 , i } } ^ { t _ { 1 } + s _ { 1 , i } } \left[\frac{\partial g}{\partial x_{i}}\left(t_{2}-s_{1, i}, \Pi_{1}\left(X_{t_{2}-s_{1, i}}\right)\right) u\left(t_{2}\right)\right.\right. \\
\left.\left.\quad-\frac{\partial g}{\partial x_{i}}\left(t_{0}, \Pi_{1}\left(X_{t_{0}}\right)\right) u\left(t_{0}+s_{1, i}\right)\right] \circ d W\left(t_{2}\right) \circ d W\left(t_{1}\right)\right\}
\end{aligned}
$$

$$
\begin{aligned}
& +\int_{t_{0}}^{t} \int_{t_{0}}^{t_{1}} \sum_{i=1}^{k_{1}} \frac{\partial g}{\partial x_{i}}\left(t_{2}, \Pi_{1}\left(X_{t_{2}}\right)\right) \bar{v}\left(t_{2}+s_{1, i}\right) d t_{2} \circ d W\left(t_{1}\right) \\
& +\sum_{i=1}^{k_{2}} \int_{t_{0}}^{t} \int_{t_{0}+s_{2, i}}^{t_{1}+s_{2, i}} \frac{\partial \bar{h}}{\partial \vec{x}_{i}}\left(t_{2}-s_{2, i}, \Pi_{2}\left(X_{t_{2}-s_{2, i}}\right)\right) u\left(t_{2}\right) \circ d W\left(t_{2}\right) d t_{1} \\
& +\int_{t_{0}}^{t} \int_{t_{0}}^{t_{1}} \sum_{i=1}^{k_{2}} \frac{\partial \bar{h}}{\partial \vec{x}_{i}}\left(t_{2}, \Pi_{2}\left(X_{t_{2}}\right)\right) \bar{v}\left(t_{2}+s_{2, i}\right) d t_{2} d t_{1}
\end{aligned}
$$

and

$$
\bar{h}:=h-\frac{1}{2} g_{k_{1}} g, \quad \bar{v}(t):= \begin{cases}\bar{h}\left(t, \Pi_{2}\left(X_{t}\right)\right), & 0 \leq t \leq a, \\ \eta(t), & t<0 .\end{cases}
$$


One can also derive the Milstein scheme for (5.24) using the StratonovichTaylor expansion (5.25) of $X(t)$ as follows. Let $t_{k}<t \leq t_{k+1}$. Then

$$
\begin{aligned}
X^{\pi}(t)= & X^{\pi}\left(t_{k}\right)+\bar{h}\left(t_{k}, \Pi_{2}\left(X_{t_{k}}^{\pi}\right)\right)\left(t-t_{k}\right) \\
& +g\left(t_{k}, \Pi_{1}\left(X_{t_{k}}^{\pi}\right)\right)\left(W(t)-W\left(t_{k}\right)\right) \\
& +\sum_{i=1}^{k_{1}} \frac{\partial g}{\partial x_{i}}\left(t_{k}, \Pi_{1}\left(X_{t_{k}}^{\pi}\right)\right) u^{\pi}\left(t_{k}+s_{1, i}\right) J\left(t_{k}+s_{1, i}, t+s_{1, i} ; s_{1, i}\right),
\end{aligned}
$$

where

$$
u^{\pi}(t)= \begin{cases}g\left(t, \Pi_{1}\left(X_{t}^{\pi}\right)\right), & t \geq 0, \\ 0, & -\tau \leq t<0 .\end{cases}
$$

5.3. The multidimensional Milstein scheme. Write $h(s, x)=\left(h^{1}(s, x), \ldots\right.$, $\left.h^{m}(s, x)\right)^{T}, \vec{x} \in \mathbf{R}^{m k_{1}}$,

$$
\vec{x}=\left(\begin{array}{ccc}
x_{11}, & \ldots, & x_{1 k_{1}} \\
\vdots & \ldots & \vdots \\
x_{m 1}, & \ldots, & x_{m k_{1}}
\end{array}\right)
$$

Denote by $g^{j l}(s, \vec{x})$ the $(j, l)$ element of the $m \times d$ matrix $g(s, \vec{x})$. To simplify notation, we use below the summation convention on repeated indices. Recall the notations for the partition $-\tau=t_{-y}<\cdots<t_{0}=0<\cdots<t_{n}=t$ introduced in Section 2. We formulate the Milstein scheme for the SDDE (1.6) as follows: if $t_{k}<t \leq t_{k+1}$, the $i$ th coordinate $X^{i}(t)$ of $X(t)=\left(X^{1}(t), \ldots, X^{m}(t)\right)^{T}$ is approximated by

$$
\begin{aligned}
X^{i, \pi}(t)=X^{i, \pi}\left(t_{k}\right)+h^{i}\left(t_{k}, \Pi_{2}\left(X_{t_{k}}^{\pi}\right)\right)\left(t-t_{k}\right) \\
+g^{i j}\left(t_{k}, \Pi_{1}\left(X_{t_{k}}^{\pi}\right)\right)\left(W^{j}(t)-W^{j}\left(t_{k}\right)\right) \\
+\frac{\partial g^{i j}}{\partial x_{i_{1} j_{1}}}\left(t_{k}, \Pi_{1}\left(X_{t_{k}}^{\pi}\right)\right) u^{i_{1} j_{1}, \pi}\left(t_{k}+s_{1, j_{1}}\right) \\
\quad \times I_{j, j_{1}}\left(t_{k}+s_{1, j_{1}}, t+s_{1, j_{1}} ; s_{1, j_{1}}\right),
\end{aligned}
$$

where

$$
u^{i_{1} j_{1}, \pi}(t)= \begin{cases}g^{i_{1} j_{1}}\left(t, \Pi_{1}\left(X_{t}^{\pi}\right)\right), & t \geq 0, \\ 0, & -\tau \leq t<0 .\end{cases}
$$

As in the SODE case $[17,18]$ and in view of Lemma 4.2, it is possible to further discretize the double stochastic integral $I_{j, j_{1}}\left(t_{k}+s_{1, j_{1}}, t+s_{1, j_{1}} ; s_{1, j_{1}}\right)$ in (5.29) 
to obtain a modified Milstein scheme for the SDDE (1.6) with strong order of convergence 1. More details are given in Appendix B.

REMARK. One may check that Lemma 5.1 and Theorems 5.2 also hold in the multidimensional case. In fact, it is easy to extend these results to the multidimensional case, thanks to the weak differentiability results (Proposition 3.1, Lemma 3.2 and Proposition 3.3) and the results concerning strong approximation of double Stratonovich integrals (Lemmas 4.1 and 4.2).

Unlike the SODE case, it seems very difficult to develop higher-order strong approximation schemes for the SDDE (1.6). One may try to avoid involving the differential operator $D$ and the trace operator $\nabla$ in the numerical scheme by attempting to employ multiple Stratonovich integrals instead of multiple Skorohod integrals. The idea is to use Stratonovich-Taylor expansions of the coefficients in the SDDE (1.6) [cf. (5.3) and (5.4)] instead of Itô-Taylor expansions. However, this is difficult, because it is hard to estimate the order of the error via the remainder term. This is because a multiple (anticipating) Stratonovich integral can not be expressed in terms of multiple (nonanticipating) Itô integrals. The $\mathrm{Hu}-$ Meyer formula gives the relationship between multiple Stratonovich and Skorohod integrals ([9], Theorem 3.1 (with nondeterministic kernels), [29], Theorem 3.1, and [27], Theorem 3.4 (with deterministic kernels)) (cf. [25, 29] and [27]). However, the formula still involves the differential operator $D$ and the trace operator $\nabla$, and hence it is hard to estimate the remainder term.

One may refer to Jolis and Sanz [16], Delgado and Sanz [9], Solé and Utzet [27] and Zakai [29] for multiple Skorohod and multiple Stratonovich integrals.

\section{APPENDIX A}

The lemma below follows from the independent increments property of Brownian motion. It is needed in the proof of the Itô formula for tame functions (Theorem 2.1).

LEMMA A.1. Assume that $\left\{\pi_{n}: 0=t_{0}<t_{1}<\cdots<t_{n}=a\right\}$ is a family of partitions of $[0, a]$, with $\lim _{n \rightarrow \infty}\left|\pi_{n}\right|=0$. Let $-\tau \leq s_{1} \leq s_{2} \leq 0$ and denote by $\Delta_{l k} W^{i}:=W^{i}\left(t_{l}+s_{k}\right)-W^{i}\left(t_{l-1}+s_{k}\right), 1 \leq i \leq \bar{d}, 1 \leq l \leq n, k=1,2$, the increments of Brownian motion. Then

$$
\lim _{n \rightarrow \infty} \sum_{l=1}^{n} \Delta_{l 1} W^{i} \Delta_{l 2} W^{j}= \begin{cases}a+s_{1}, & \text { if } i=j \text { and } s_{1}=s_{2} \\ 0, & \text { otherwise }\end{cases}
$$

in $L^{2}(\Omega, \mathbf{R})$. 
PROOF. We only need to consider the cases $s_{1}<s_{2}$ and $i=j$. Now

$$
\begin{aligned}
& {\left[\sum_{l=1}^{n} \Delta_{l 1} W^{i} \Delta_{l 2} W^{i}\right]^{2}} \\
& \quad=\sum_{l=1}^{n}\left(\Delta_{l 1} W^{i}\right)^{2}\left(\Delta_{l 2} W^{i}\right)^{2}+2 \sum_{l_{1}<l_{2}} \Delta_{l_{1} 1} W^{i} \Delta_{l_{1} 2} W^{i} \Delta_{l_{2} 1} W^{i} \Delta_{l_{2} 2} W^{i} .
\end{aligned}
$$

If $n$ is sufficiently large, then $\left|\pi_{n}\right|<s_{2}-s_{1}$. Hence $\Delta_{l_{2} 2} W^{i}$ is independent of $\Delta_{l_{1} 1} W^{i} \Delta_{l_{1} 2} W^{i} \Delta_{l_{2} 1} W^{i}$. Taking expectations in the above equality gives

$$
E\left[\sum_{l=1}^{n} \Delta_{l 1} W^{i} \Delta_{l 2} W^{i}\right]^{2} \leq \sum_{l=1}^{n}\left(t_{l}-t_{l-1}\right)^{2} \leq\left|\pi_{n}\right| a
$$

for sufficiently large $n$. Note that $a+s_{1}$ is the correct limit in (2.6) because of the convention that $W(t)=0$ for $t<0$. This completes the proof of the lemma.

The following lemma extends a result by Nualart and Pardoux ([23], Lemma C1).

LEMMA A.2. Suppose that $x=\{x(t): t \in[0, a]\}$ is a measurable real-valued process, $x(t)=0$ if $t>a$ or $t<0$, and $x \in L^{p}([0, a], \mathbf{R})$ a.s., $p>1$. Assume that $\left\{\pi_{n}: 0=t_{0}<t_{1}<\cdots<t_{n}=a\right\}$ is a family of partitions of $[0, a]$, with $\lim _{n \rightarrow \infty}\left|\pi_{n}\right|=0$, and $-\tau \leq s_{1}, s_{2} \leq 0$. Then

$$
\lim _{n \rightarrow \infty} \sum_{l=1}^{n} \frac{\Delta_{l 1} W \Delta_{l 2} W}{t_{l}-t_{l-1}} \int_{t_{l-1}+s_{1}}^{t_{l}+s_{1}} x(s) d s= \begin{cases}\int_{0}^{a+s_{1}} x(s) d s, & s_{1}=s_{2}, \\ 0, & s_{1} \neq s_{2},\end{cases}
$$

in probability. Moreover, if $x \in L^{p}(\Omega \times[0, a], \mathbf{R})$, then the above convergences hold in $L^{1}(\Omega, \mathbf{R})$.

PROOF. It clearly suffices to show that (A.2) holds in $L^{1}(\Omega, \mathbf{R})$ whenever $x \in L^{p}(\Omega \times[0, a], \mathbf{R})$. Fix $m \geq 1$, define

$$
x^{m}:=\sum_{l=1}^{m} \frac{I_{\left(t_{l-1}+s_{1}, t_{l}+s_{1}\right]}}{t_{l}-t_{l-1}} \int_{t_{l-1}+s_{1}}^{t_{l}+s_{1}} x(s) d s .
$$

For $n \geq 1$, define

$$
\alpha_{n}(x):=\sum_{l=1}^{n} \frac{\Delta_{l 1} W \Delta_{l 2} W}{t_{l}-t_{l-1}} \int_{t_{l-1}+s_{1}}^{t_{l}+s_{1}} x(s) d s .
$$

Define $\alpha_{n}\left(X_{m}\right)$ similarly. It follows from Hölder's inequality that if $1 / p+1 / q=1$, then

$$
E\left|\alpha_{n}(x)\right| \leq\left\{E \sum_{l=1}^{n} \frac{\left|\Delta_{l 1} W \Delta_{l 2} W\right|^{q}}{\left(t_{l}-t_{l-1}\right)^{q-1}}\right\}^{1 / q}\left\{E \sum_{l=1}^{n} \frac{\left(\int_{t_{l-1}+s_{1}}^{t_{l}+s_{1}}|x(s)| d s\right)^{p}}{\left(t_{l}-t_{l-1}\right)^{p / q}}\right\}^{1 / p}
$$


that is

$$
\left\|\alpha_{n}(x)\right\|_{L^{1}(\Omega)} \leq C_{p}\|x\|_{L^{p}\left(\Omega \times\left[0, a+s_{1}\right]\right)} \leq C_{p}\|x\|_{L^{p}(\Omega \times[0, a])} .
$$

Therefore,

$$
\begin{aligned}
& E\left|\alpha_{n}(x)-\int_{0}^{a+s_{1}} x(s) d s\right| \\
& \quad \leq E\left|\alpha_{n}\left(x-x^{m}\right)\right|+E\left|\alpha_{n}\left(x^{m}\right)-\int_{0}^{a+s_{1}} x(s) d s\right| \\
& \quad \leq E\left|\alpha_{n}\left(x^{m}\right)-\int_{0}^{a+s_{1}} x(s) d s\right|+C_{p}\left\|x-x^{m}\right\|_{L^{p}\left(\Omega \times\left[0, a+s_{1}\right]\right)}
\end{aligned}
$$

since

$$
\begin{aligned}
\alpha_{n}\left(x^{m}\right)= & \sum_{i=1}^{m}\left\{\sum_{\left.t_{l-1}, t_{l}\right] \subseteq\left(t_{i-1}, t_{i}\right], 1 \leq l \leq n} \int_{t_{i-1}+s_{1}}^{t_{i}+s_{1}} \frac{I_{\left(t_{i-1}+s_{1}, t_{i}+s_{1}\right]}(t)}{t_{l}-t_{l-1}} d t \Delta_{l 1} W \Delta_{l 2} W\right\} \\
& \times \frac{1}{t_{i}-t_{i-1}} \int_{t_{i-1}+s_{1}}^{t_{i}+s_{1}} x(s) d s \\
= & \sum_{i=1}^{m}\left\{\sum_{\left.t_{l-1}, t_{l}\right] \subseteq\left(t_{i-1}, t_{i}\right], 1 \leq l \leq n} \Delta_{l 1} W \Delta_{l 2} W\right\} \frac{1}{t_{i}-t_{i-1}} \int_{t_{i-1}+s_{1}}^{t_{i}+s_{1}} x(s) d s .
\end{aligned}
$$

Let $k_{m}$ be the index such that $t_{k_{m}-1}+s_{1}<0 \leq t_{k_{m}}+s_{1}$. If $s_{1}=s_{2}$, then by Lemma A.1, the following limit exists in probability:

$$
\begin{aligned}
\lim _{n \rightarrow \infty} \alpha_{n}\left(x^{m}\right) & =\sum_{i=1}^{m}\left[\left(t_{i}+s_{1}\right) \wedge 0-\left(t_{i-1}+s_{1}\right) \vee 0\right] \frac{1}{t_{i}-t_{i-1}} \int_{t_{i-1}+s_{1}}^{t_{i}+s_{1}} x(s) d s \\
& =\sum_{i=k_{m}+1}^{m} \int_{t_{i-1}+s_{1}}^{t_{i}+s_{1}} x(s) d s+\frac{t_{k_{m}}+s_{1}}{t_{k_{m}-t_{k_{m}-1}}} \int_{0}^{t_{k_{m}}+s_{1}} x(s) d s \\
& =\int_{0}^{a+s_{1}} x(s) d s+\frac{t_{k_{m}}+s_{1}}{t_{k_{m}-t_{k_{m}-1}}} \int_{0}^{t_{k_{m}}+s_{1}} x(s) d s .
\end{aligned}
$$

Equivalently,

$$
\alpha_{n}\left(x^{m}\right)-\int_{0}^{a+s_{1}} x(s) d s-\frac{t_{k_{m}}+s_{1}}{t_{k_{m}-t_{k_{m}-1}}} \int_{0}^{t_{k_{m}}+s_{1}} x(s) d s \rightarrow 0
$$

as $n \rightarrow \infty$ in probability.

A slight modification in the proof of (A.3) yields the estimate

$$
\left\|\alpha_{n}\left(x^{m}\right)\right\|_{L^{p^{\prime}}(\Omega)} \leq C\left(p, p^{\prime}\right)\left\|x^{m}\right\|_{L^{p}\left(\Omega \times\left[0, a+s_{1}\right]\right)},
$$


for all $p^{\prime} \in(1, p)$. Therefore, the family $\left\{\alpha_{n}\left(x^{m}\right): n \geq 1\right\}$ is uniformly integrable. From (A.4) we have

$$
\begin{aligned}
\lim _{n \rightarrow \infty} & E\left|\alpha_{n}(x)-\int_{0}^{a+s_{1}} x(s) d s\right| \\
& \leq E\left|\frac{t_{k_{m}}+s_{1}}{t_{k_{m}-t_{k_{m}-1}}} \int_{0}^{t_{k_{m}}+s_{1}} x(s) d s\right|+C_{p}\left\|x-x^{m}\right\|_{L^{p}\left(\Omega \times\left[0, a+s_{1}\right]\right)} \\
& \leq E \int_{0}^{t_{k_{m}}+s_{1}}|x(s)| d s+C_{p}\left\|x-x^{m}\right\|_{L^{p}\left(\Omega \times\left[0, a+s_{1}\right]\right)} .
\end{aligned}
$$

Clearly, $x^{m} \rightarrow x$ in $L^{p}\left(\Omega \times\left[0, a+s_{1}\right], \mathbf{R}\right)$ and $E \int_{0}^{t_{k_{m}}+s_{1}}|x(s)| d s \rightarrow 0$ as $m \rightarrow \infty$. So

$$
\lim _{n \rightarrow \infty} E\left|\alpha_{n}\left(x^{m}\right)-\int_{0}^{a+s_{1}} x(s) d s\right|=0 .
$$

Now consider the case $s_{1} \neq s_{2}$. Since

$$
\begin{aligned}
E\left|\alpha_{n}(x)\right| & \leq E\left|\alpha_{n}\left(x^{m}\right)\right|+E\left|\alpha_{n}\left(x-x^{m}\right)\right| \\
& \leq E\left|\alpha_{n}\left(x^{m}\right)\right|+C_{p}\left\|x-x^{m}\right\|_{L^{p}\left(\Omega \times\left[0, a+s_{1}\right]\right)},
\end{aligned}
$$

a similar argument gives $\lim _{n \rightarrow \infty} E\left|\alpha_{n}(x)\right|=0$.

The following useful result is due to Föllmer [10], and Nualart and Pardoux ([23], Lemma C.2).

LEMMA A.3. Let $x^{i}(t), 0 \leq t \leq a, i=1,2$, be two-continuous processes, and $\left\{\pi_{n}: 0=t_{0}<t_{1}<\cdots<t_{n}=a\right\}$ a family of partitions of $[0, a]$, with $\lim _{n \rightarrow \infty}\left|\pi_{n}\right|=0$. For each $n$ and $l=1, \ldots, n$, let $x_{t_{l}, n}^{i}$ denote $x^{i}\left(t_{l}\right)$. Assume that

$$
\sum_{l=1}^{n}\left(x_{t_{l}, n}^{i}-x_{t_{l-1}, n}^{i}\right)\left(x_{t_{l}, n}^{j}-x_{t_{l-1}, n}^{j}\right) \rightarrow \int_{0}^{a} a^{i j}(s) d s
$$

in probability as $n \rightarrow \infty$, where $\left\{a^{i j}(t): 0 \leq t \leq a ; i, j=1,2\right\}$ are measurable processes such that a.s.

$$
\int_{0}^{a}\left|a^{i j}(s)\right| d s<\infty, \quad i, j=1,2 .
$$

Let $\{Y(t): 0 \leq t \leq a\}$ be a continuous process, and $\left\{Y^{n}(t): 0 \leq t \leq a\right\}_{n=1}^{\infty}$ be measurable processes which converge a.s. to $\{Y(t)\}$ as $n \rightarrow \infty$, uniformly with respect to $t \in[0, a]$. Then

$$
\sum_{l=1}^{n} Y^{n}\left(t_{l-1}\right)\left(x_{t_{l}, n}^{i}-x_{t_{l-1}, n}^{i}\right)\left(x_{t_{l}, n}^{j}-x_{t_{l-1}, n}^{j}\right) \rightarrow \int_{0}^{a} a^{i j}(s) Y(s) d s
$$

in probability as $n \rightarrow \infty$, for $i=1,2$. 


\section{APPENDIX B}

Simulating a double stochastic integral. The following scheme is adapted from Kloeden and Platen ([17], page 202, and [18], page 82).

In view of Lemma 4.2, we can use the truncated sums

$$
\begin{aligned}
J_{i, j}^{p}(0, t ;-b)= & \frac{1}{2}\left(W^{i}(t) B^{j}(t)\right)-\frac{1}{2}\left(W^{i}(t) a_{0}^{j, b}-B^{j}(t) a_{0}^{i}\right) \\
& +\pi \sum_{n=1}^{p} n\left(a_{n}^{i} b_{n}^{j, b}-b_{n}^{i} a_{n}^{j, b}\right), \quad t \geq 0, p \geq 1
\end{aligned}
$$

to simulate the double Stratonovich integral

$$
J_{i, j}(0, t ;-b)=\int_{b}^{t+b} \int_{0}^{s-b} \circ d W^{i}(v) \circ d W^{j}(s) .
$$

Consider the Milstein scheme (5.9). Given an error bound $\delta=O\left(|\pi|^{2}\right)$, we choose an integer $p \geq 1$ such that

$$
\frac{1}{p} \leq \delta \wedge \min \left\{\left|s_{1, i}\right|: 1 \leq i \leq k_{1}\right\} .
$$

We define for all such integers $p$,

$$
I^{p}\left(t_{k}+s_{1, i}, t+s_{1, i} ; s_{1, i}\right):= \begin{cases}J_{i j}^{p}\left(t_{k}+s_{1, i}, t+s_{1, i} ; s_{1, i}\right), & s_{1, i}<0, \\ \frac{\left(W(t)-W\left(t_{0}\right)\right)^{2}}{2}-\frac{t-t_{0}}{2}, & s_{1, i}=0 .\end{cases}
$$

By Lemma 4.2, the following modification of the Milstein scheme:

$$
\begin{array}{r}
X^{\pi}(t)=X^{\pi}\left(t_{k}\right)+h\left(t_{k}, \Pi_{2}\left(X_{t_{k}}^{\pi}\right)\right)\left(t-t_{k}\right)+g\left(t_{k}, \Pi_{1}\left(X_{t_{k}}^{\pi}\right)\right)\left(W(t)-W\left(t_{k}\right)\right) \\
+\sum_{i=1}^{k_{1}} \frac{\partial g}{\partial x_{i}}\left(t_{k}, \Pi_{1}\left(X_{t_{k}}^{\pi}\right)\right) u^{\pi}\left(t_{k}+s_{1, i}\right) I^{p}\left(t_{k}+s_{1, i}, t+s_{1, i} ; s_{1, i}\right), \\
t_{k}<t \leq t_{k+1},
\end{array}
$$

has strong order of convergence 1 (cf. Theorem 5.2).

Suppose that we use the family of partitions: $\pi:-1=t_{-l}<\cdots<t_{0}=0<$ $\cdots<t_{n}=a, \operatorname{mesh} \pi=|\pi|$, to calculate the solution of the $\operatorname{SDDE}$ (1.6) (with $\tau=1$ ) by applying the Milstein scheme. There are some issues we need to consider concerning simulating the family

$$
S=\left\{J\left(t_{k-1}, t_{k} ; s_{1, i}\right): k=1, \ldots, n, i=1, \ldots, k_{1}\right\} .
$$

If $k_{1} \neq k_{2}$ or $i_{1} \neq i_{2}$, by the Itô isometry, $J\left(t_{k_{1}-1}, t_{k_{1}} ; s_{1, i_{1}}\right)$ and $J\left(t_{k_{2}-1}, t_{k_{2}} ; s_{1, i_{2}}\right)$ are independent. But the family $S$ may not be independent. The reason is that they come from the same Brownian motion. We can make $S$ independent by choosing appropriate mesh points so that $t_{k}+s_{1, i} \in \pi$, for all $k \geq 0,1 \leq i \leq k_{1}$; that is, 
$t_{k}+s_{1, i}$ is also a mesh point. In order to see this, set

$$
V_{k}=\left\{\left(a_{n}\left(t_{k-1}\right), b_{n}\left(t_{k-1}\right)\right): n=0,1, \ldots\right\},
$$

where $a_{n}\left(t_{k-1}\right)$ and $b_{n}\left(t_{k-1}\right)$ are defined by (4.6). Then for all $1 \leq i \leq k_{1}$, $1 \leq k \leq n$, the set

$$
V_{k}\left(s_{1, i}\right)=\left\{\left(a_{n}^{-s_{1, i}}\left(t_{k-1}\right), b_{n}^{-s_{1, i}}\left(t_{k-1}\right)\right): n=0,1, \ldots\right\}
$$

belongs to the family $\left\{V_{k}: k=1, \ldots, n\right\}$, where $a_{n}^{-s_{1, i}}\left(t_{k-1}\right)$ and $b_{n}^{-s_{1, i}}\left(t_{k-1}\right)$ are defined by (4.7). Indeed, similar to the approximation scheme of multiple Stratonovich integrals ([17], (5.8.10) and (5.8.12), [18], (2.3.30) and (2.3.32)), we have the following approximation scheme of $\left\{J^{p}\left(t_{k-1}, t_{k} ; s_{1, i}\right): k=1, \ldots, n\right.$, $\left.i=1, \ldots, k_{1}\right\}, p \geq 1$.

For each $k=1, \ldots, n$, and $h=1, \ldots, p$, with $p \geq 1$, we define $\rho_{p}$ and independent $N(0,1)$ random variables $\xi(s), \mu_{p}(s), \zeta_{h}(s), \eta_{h}(s), s \in\left\{t_{0}, \ldots, t_{n-1}\right\}$, by

$$
\begin{aligned}
\xi(s) & =\frac{1}{|\pi|}(W(|\pi|+s)-W(s)), \\
\zeta_{h}(s) & =\sqrt{\frac{2}{|\pi|}} h \pi a_{h}(s), \quad \eta_{h}(s)=\sqrt{\frac{2}{|\pi|} h \pi b_{h}(s),} \\
\rho_{p} & =\frac{1}{12}-\frac{1}{2 \pi^{2}} \sum_{h=1}^{p} \frac{1}{h^{2}}, \quad \mu_{p}(s)=\frac{1}{\sqrt{|\pi| \rho_{p}}} \sum_{h=p+1}^{\infty} a_{h}(s), \\
a_{0}(s) & =-\frac{1}{\pi} \sqrt{2|\pi|} \sum_{h=1}^{p} \frac{1}{h} \zeta_{h}(s)-2 \sqrt{|\pi| \rho_{p}} \mu_{p}(s) .
\end{aligned}
$$

If $t_{k-1}+s_{1, i} \geq 0$, then

$$
\begin{aligned}
J^{p}\left(t_{k-1}\right. & \left.+s_{1, i}, t_{k}+s_{1, i} ; s_{1, i}\right) \\
= & \frac{1}{2}|\pi| \xi\left(t_{k-1}+s_{1, i}\right) \xi\left(t_{k-1}\right) \\
& -\frac{1}{2} \sqrt{|\pi|}\left[\xi\left(t_{k-1}+s_{1, i}\right) a_{0}\left(t_{k-1}\right)-\xi\left(t_{k-1}\right) a_{0}\left(t_{k-1}+s_{1, i}\right)\right] \\
& +\frac{|\pi|}{2 \pi} \sum_{h=1}^{p} \frac{1}{h}\left[\zeta_{h}\left(t_{k-1}+s_{1, i}\right) \eta_{h}\left(t_{k-1}\right)-\zeta_{h}\left(t_{k-1}\right) \eta_{h}\left(t_{k-1}+s_{1, i}\right)\right] .
\end{aligned}
$$

REMARKS.

1. The space complexity of the Milstein scheme for an SDDE is $O(m a /|\pi|)$ if we only want to simulate the end point $X(a)$ (or the end segment $X_{a}$ ). The space 
complexity of the Milstein scheme for an $m$-dimensional SODE is $O(m a)$ if we only want to simulate the end point $X(a)$. If we want to simulate the whole path $\{X(t): t \in[0, a]\}$, then both schemes have the same space complexity $O(m a /|\pi|)$.

2. Roughly speaking, the time complexity of the Milstein scheme for a multidimensional SDDE $(m>1)$ is $K$ times the time complexity of the corresponding scheme for an SODE, where $K:=k_{1}+k_{2}$ is the total number of delays. If $m=1$, we can directly simulate the double stochastic integral in the Milstein scheme using (B.3).

3. In view of (B.7) and (B.8), we do not need to simulate the joint law of multivariate normal variables for multidimensional SDDEs and SODEs. If $m$ is not very large, simulating the joint law is not a prohibitive task by using Cholesky's decomposition.

Acknowledgments. The authors are very grateful to the anonymous referees and to the editors of the Annals of Probability for their helpful suggestions and comments on an earlier version of the manuscript.

\section{REFERENCES}

[1] Ahmed, T. A. (1983). Stochastic functional differential equations with discontinuous initial data. M.Sc. thesis, Univ. Khartoum, Sudan.

[2] Alòs, E. and NuALART, D. (1998). An extension of Itô's formula for anticipating processes. J. Theoret. Probab. 2 493-514.

[3] Asch, J. and PоттноғF, J. (1991). Itô's lemma without non-anticipatory conditions. Probab. Theory Related Fields 88 17-46.

[4] BAKER, C. T. H. and BUCKWAR, E. (2000). Numerical analysis of explicit one-step methods for stochastic delay differential equations. LMS J. Comput. Math. 3 315-335 (electronic). Available at www.lms.ac.uk/jcm.

[5] Bell, D. and Mohammed, S.-E. A. (1989). On the solution of stochastic ordinary differential equations via small delays. Stochastics Stochastics Rep. 28 293-299.

[6] Bell, D. and Mohammed, S.-E. A. (1991). The Malliavin calculus and stochastic delay equations. J. Funct. Anal. 99 75-99.

[7] Berger, M. and Mizel, V. J. (1982). An extension of the stochastic integral. Ann. Probab. 10 435-450.

[8] Cambanis, S. and Hu, Y. (1996). The exact convergence rate of Euler-Maruyama scheme and application to sample design. Stochastics Stochastics Rep. 59 211-240.

[9] Delgado, R. and Sanz, M. (1992). The Hu-Meyer formula for non-deterministic kernels. Stochastics Stochastics Rep. 38 149-158.

[10] Föllmer, H. (1981). Calcul d'Itô sans probabilités. Seminar en Probability XV. Lecture Notes in Math. 850 143-150. Springer, New York.

[11] Gaines, J. G. and LyOns, T. J. (1994). Random generation of stochastic area integrals. SIAM J. Appl. Math. 54 1132-1146.

[12] Gentle, J. (1998). Random Number Generation and Monte Carlo Methods. Springer, New York.

[13] HU, Y. (1996). Strong and weak order of time discretization schemes of stochastic differential equations. Séminaire de Probabilités XXX. Lecture Notes in Math. 1626 218-227. Springer, Berlin. 
[14] Hu, Y. (2000). Optimal times to observe in the Kalman-Bucy model. Stochastics Stochastic Rep. 69 123-140.

[15] Hu, Y., Mohammed, S.-E. A. and Yan, F. (2001). Discrete-time approximations of stochastic differential systems with memory. Dept. Mathematics, Southern Illinois Univ., Carbondale. Available at http://sfde.math.siu.edu/recentpub.html.

[16] JoLIs, M. and SANZ, M. (1988). On generalized multiple stochastic integrals and multiparameter anticipative calculus. Stochastic Analysis and Related Topics II. Lecture Notes in Math. 1444 141-182. Springer, New York.

[17] Kloeden, P. and Platen, E. (1992). Numerical Solution of Stochastic Differential Equations. Springer, New York.

[18] Kloeden, P., Platen, E. and Schurz, H. (1994). Numerical Solution of SDE Through Computer Experiments. Springer, Berlin.

[19] McShane, E. J. (1974). Stochastic Calculus and Stochastic Models. Academic Press, New York.

[20] Mohammed, S.-E. A. (1984). Stochastic Functional Differential Equations. Research Notes in Mathematics 99. Pitman Books, London.

[21] Mohammed, S.-E. A. (1998). Stochastic differential systems with memory: Theory, examples and applications. In Stochastic Analysis and Related Topics VI (L. Decreusefond, J. Gjerde, B. Øksendal and A. S. Ustunel, eds.) 1-77. Birkhäuser, Boston.

[22] Nualart, D. (1995). The Malliavin Calculus and Related Topics. Springer, New York.

[23] Nualart, D. and Pardoux, E. (1988). Stochastic calculus with anticipating integrands. Probab. Theory Related Fields $\mathbf{7 8} 535-581$.

[24] Pardoux, E. and Protter, P. (1990). Stochastic Volterra equations with anticipating coefficients. Ann. Probab. 18 1635-1655.

[25] Russo, F. and VAllois, P. (1993). Forward, backward and symmetric stochastic integration. Probab. Theory Related Fields 97 403-421.

[26] Ryden, T. and Wiktorsson, M. (2001). On the simulation of iterated Itô integrals. Stochastic Process. Appl. 91 151-168.

[27] Solé, J. and UtZet, F. (1990). Stratonovich integral and trace. Stochastics Stochastics Rep. $29203-220$.

[28] Yan, F. (1999). Topics on stochastic delay equations. Ph.D. dissertation, Southern Illinois Univ., Carbondale, Illinois.

[29] ZaKaI, M. (1990). Stochastic integration, trace and the skeleton of Wiener functionals. Stochastics Stochastics Rep. 32 93-108.

Y. HU

DEPARTMENT OF MATHEMATICS

UNIVERSITY OF KANSAS

LAWRENCE, KANSAS 66045-2142

USA

E-MAIL:hu@math.ukans.edu

\author{
S.-E. A. Mohammed \\ DEPARTMENT OF MATHEMATICS \\ SOUTHERN ILLINOIS UNIVERSITY \\ CARBONDALE, ILLINOIS 62901 \\ USA \\ E-MAIL: salah@sfde.math.siu.edu
}

\author{
F. YAN \\ WiLliams ENERGY MARKETING \\ AND TRADING \\ ONE Williams CENTER, WRC2-4 \\ TULSA, OKLAHOMA 74119 \\ USA \\ E-MAIL: fyan1@yahoo.com
}

